\title{
Probabilistic Belief Logics for Uncertain Agents
}

\author{
Zining $\mathrm{CaO}^{1,2}$ \\ ${ }^{1}$ Department of Computer Science and Technology, Nanjing Universityof Aero. $\mathcal{E}$ Astro., \\ Nanjing \\ 2Provincial Key Laboratory for Computer Information Processing Technology, \\ Soochow University, Suzhou \\ P. R. China
}

\section{Introduction}

The study of knowledge and belief has a long tradition in philosophy. An early treatment of a formal logical analysis of reasoning about knowledge and belief came from Hintikka's work [15]. More recently, researchers in such diverse fields as economics, linguistics, artificial intelligence and theoretical computer science have become increasingly interested in reasoning about knowledge and belief $[1-5,10-13,18,20,24]$. In wide areas of application of reasoning about knowledge and belief, it is necessary to reason about uncertain information. Therefore the representation and reasoning of probabilistic information in belief is important.

There has been a lot of works in the literatures related to the representation and reasoning of probabilistic information, such as evidence theory [25], probabilistic logic [4], probabilistic dynamic logic [7], probabilistic nonmonotonic logic [21], probabilistic knowledge logic [3] and etc. A distinguished work is done by Fagin and Halpern [3], in which a probabilistic knowledge logic is proposed. It expanded the language of knowledge logic by adding formulas like " $w_{i}(\varphi) \geq 2 w_{i}(\psi)$ " and " $w_{i}(\varphi)<1 / 3$ ", where $\varphi$ and $\psi$ are arbitrary formulas. These formulas mean " $\varphi$ is at least twice probable as $\psi$ " and " $\varphi$ has probability less than $1 / 3$ ". The typical formulas of their logic are " $a_{1} w_{i}\left(\varphi_{1}\right)+\ldots+a_{k} w_{i}\left(\varphi_{k}\right) \geq b^{\prime \prime}$, " $K_{i}(\varphi)$ " and " $K_{i}^{b}(\varphi)$ ", the latter formula is an abbreviation of " $K_{i}\left(w_{i}(\varphi) \geq b\right)$ ". Here formulas may contain nested occurrences of the modal operators $w_{i}$ and $K_{i}$, and the formulas in [4] do not contain nested occurrences of the modal operators $w_{i}$. On the basis of knowledge logic, they added axioms of reasoning about linear inequalities and probabilities. To provide semantics for such logic, Fagin and Halpern introduced a probability space on Kripke models of knowledge logic, and gave some conditions about probability space, such as $O B J, S D P$ and UNIF. At last, Fagin and Halpern concluded by proving the soundness and completeness of their probabilistic knowledge logic.

Fagin and Halpern's work on probabilistic epistemic logic is well-known and original. However, there are several aspects worth further investigation: First, the completeness proof of Fagin and Halpern can only deal with the finite set of formulas for that their method reduces the completeness to the existence of a solution of a set of finitely many linear inequalities. In the case of an infinite set of formulas, their method reduces the problem to the existence of a solution of infinitely many linear inequalities with infinitely many variables, which does not seem to be captured by the axioms in [3] for their language only contains finite-length 
formulas. Second, their inference system includes axioms about linear inequalities and probabilities, which makes the system complicated. Third, the semantics in [3] was given by adding a probability space on Kripke structure, correspondingly there are restrictions on probability spaces and accessible relations, but in fact a simpler model is possible for the semantics of probabilistic epistemic logic.

Kooi's work [18] combines the probabilistic epistemic logic with the dynamic epistemic logic yielding a new logic, $P D E L$, that deals with changing probabilities and takes higher-order information into account. The syntax of PDEL is an expansion of Fagin and Halpern's logic by introducing formula " $\left[\varphi_{1}\right] \varphi_{2}$ ", which can be read as " $\varphi_{2}$ is the case, after everyone simultaneously and commonly learns that $\varphi_{1}$ is the case". The semantics of PDEL is essentially same as Fagin and Halpern's semantics, which is based on a combination of Kripke structure and probability functions. Kooi proved the soundness and weak completeness of $P D E L$, but like Fagin and Halpern's paper, completeness of PDEL was not given.

In [22], the authors also propose a probabilistic belief logic, called PEL, which is essentially a restricted version of the logic proposed by Fagin and Halpern. But in this chapter, the inference system was not given and the corresponding properties such as soundness and completeness of PEL were not studied.

In [16], Hoek investigated a probabilistic logic $P_{F} D$. This logic is enriched with operators $P_{r}^{>},(r \in[0,1])$ where the intended meaning of $P_{r}^{>} \varphi$ is "the probability of $\varphi$ is strictly greater than $r^{\prime \prime}$. The author gave a completeness proof of $P_{F} D$ by the construction of a canonical model for $P_{F} D$ considerably. Furthermore, the author also proved finite model property of the logic by giving a filtration-technique for the intended models. Finally, the author prove the decidability of the logic. In [16], the logic $P_{F} D$ is based on a set $F$, where $F$ is a finite set and $\{0,1\} \subseteq F \subseteq[0,1]$. The completeness of $P_{F} D$ was not proved in [16] for the case that $F$ is infinite. Hoek presented this problem as an open question and considered it as a difficult task. He think this problem may be tackled by introducing infinitary rules.

In this chapter, we propose some probabilistic belief logics. There is no axiom and rule about linear inequalities and probabilities in the inference system of probabilistic belief logics. Hence the inference system looks simpler and uniform than Fagin and Halpern's logic. We also propose a simpler semantics for probabilistic belief logics, where is no accessible relation and can be generalized to description semantics of other probabilistic modal logics. Moreover, we present the new completeness proofs for our probabilistic belief logics, which can deal with infinite sets of formulas.

The remainder of the chapter is organized as follows: In Section 2, we propose a probabilistic belief logic, called $P B L_{\omega}$. We provide the probabilistic semantics of $P B L_{\omega}$, and prove the soundness and completeness of $P B L_{\omega}$ with respect to the semantics. We are unable to prove or disprove the finite model property of $P B L_{\omega}$ in this chapter even though we conjecture it holds. We turn to look at a variant of $P B L_{\omega}$, which has the finite model property. In Section 3, we present a weaker variant of $P B L_{\omega}$, called $P B L_{f}$, which is the same as $P B L_{\omega}$ but without Axiom 6 and Rule 6. We give the semantics of $P B L_{f}$ and prove the soundness and finite model property of $P B L_{f}$. As a consequence, the weak completeness of $P B L_{f}$ is given, i.e., for any finite set of formulas $\Gamma, \Gamma \models{ }_{P B L_{f}} \varphi \Rightarrow \Gamma \vdash_{P B L_{f}} \varphi$. But there is an infinite inference rule, namely Rule 5, in $P B L_{f}$, which is inconvenient for application. Therefore we consider another variant $P B L_{r}$ in Section 4 . The axioms and rules of $P B L_{r}$ are same as $P B L_{f}$ except for Rule 5. $P B L_{r}$ has a syntax restriction that the probability $a$ in the scope of $B_{i}(a, \varphi)$ must be a 
rational number. The soundness and finite model property of $P B L_{r}$ are proved. From the finite model property, we obtain the weak completeness of $P B L_{r}$. Note that a logic system has the compactness property if and only if the weak completeness is equivalent to the completeness in that logic. The compactness property does not hold in $P B L_{r}$, for example, $\left\{B_{i}(1 / 2, \varphi)\right.$, $\left.B_{i}(2 / 3, \varphi), \ldots, B_{i}(n / n+1, \varphi), \ldots\right\} \cup\left\{\neg B_{i}(1, \varphi)\right\}$ is not satisfied in any $P B L_{r}$-model, but any finite subset of it has a model. Therefore the weak completeness of $P B L_{r}$ is not equivalent to the completeness. $P B L_{r}$ is proved to be weak complete. Furthermore, the decidability of $P B L_{r}$ is shown. In Section 5, we mainly compare our logics with the logic in [3] in terms of their syntax, inference system, semantics and proof technique. The chapter is concluded in Section 6.

\section{2. $P B L_{\omega}$ and its probabilistic semantics}

In this section, we first review the standard belief logic system and the standard Kripke semantics. Some examples are given to illustrate why it is necessary to extend belief to probabilistic belief. Then we introduce a probabilistic belief logic $P B L_{\omega}$.

In belief logic, the formula $B_{i} \varphi$ says that agent $i$ believes $\varphi$. Consider a system with $n$ agents, say $1, \ldots, n$, and we have a nonempty set $\Phi$ of primitive propositions about which we wish to reason. We construct formulas by closing off $\Phi$ under conjunction, negation and modal operators $B_{i}$, for $i=1, \ldots, n$ (where $B_{i} \varphi$ is read as "agent $i$ believes $\varphi^{\prime \prime}$ ).

The semantics to these formulas is given by means of Kripke structure [19]. A Kripke structure for belief (for $n$ agents) is a tuple $\left(S, \pi, R_{1}, \ldots, R_{n}\right)$, where $S$ is a set of states, $\pi(s)$ is a truth assignment to the primitive propositions of $\Phi$ for each state $s \in S$, and $R_{i}$ is an accessible relation on $S$, which satisfies the following conditions: Euclideanness $\left(\forall s \forall s^{\prime} \forall s^{\prime \prime}\left(s R_{i} s^{\prime} \wedge s R_{i} s^{\prime \prime} \rightarrow s^{\prime} R_{i} s^{\prime \prime}\right)\right)$, transitivity $\left(\forall s \forall s^{\prime} \forall s^{\prime \prime}\left(s R_{i} s^{\prime} \wedge s^{\prime} R_{i} s^{\prime \prime} \rightarrow s R_{i} s^{\prime \prime}\right)\right)$ and definality $\left(\forall s \exists s^{\prime}\left(s R_{i} s^{\prime}\right)\right)$.

We now assign truth values to formulas at each state in the structure. We write $(M, s) \models \varphi$ if the formula $\varphi$ is true at state $s$ in Kripke structure $M$.

$$
\begin{aligned}
& (M, s) \models p \text { (for } p \in \Phi) \text { iff } \pi(s)(p)=\text { true } \\
& (M, s) \models \neg \varphi \text { iff }(M, s) \not \models \varphi \\
& (M, s) \models \varphi \wedge \psi \text { iff }(M, s) \models \varphi \text { and }(M, s) \models \psi \\
& (M, s) \models B_{i} \varphi \text { iff }(M, t) \models \varphi \text { for all } t \in R_{i}(s) \text { with } R_{i}(s)=\left\{s^{\prime} \mid\left(s, s^{\prime}\right) \in R_{i}\right\}
\end{aligned}
$$

The last clause in this definition captures the intuition that agent $i$ believes $\varphi$ in world $(M, s)$ exactly if $\varphi$ is true in all worlds that $i$ considers possible.

It is well known that the following set of axioms and inference rules provides a sound and complete axiomatization for the logic of belief with respect to the class of Kripke structures for belief:

All instances of propositional tautologies and rules.

$$
\begin{aligned}
& \left(B_{i} \varphi \wedge B_{i}(\varphi \rightarrow \psi)\right) \rightarrow B_{i} \psi \\
& B_{i} \varphi \rightarrow \neg B_{i} \neg \varphi \\
& B_{i} \varphi \rightarrow B_{i} B_{i} \varphi
\end{aligned}
$$


$\neg B_{i} \varphi \rightarrow B_{i} \neg B_{i} \varphi$

$\vdash \varphi \Rightarrow \vdash B_{i} \varphi$

There are examples of probabilistic belief in daily life. For example, one may believe that the probability of "it will rain tomorrow" is less than 0.4 ; in a football game, one may believe that the probability of "team $A$ will win" is no less than 0.7 and so on. In distribute systems, there may be the cases that "agent $i$ believes that the probability of 'agent $j$ believes that the probability of $\varphi$ is at least $a^{\prime}$ is no less than $b^{\prime \prime}$. Suppose there are two persons communicating by email, agent $A$ sends an email to agent $B$. Since the email may be lost in network, $A$ does not know whether $B$ has received the email. Therefore $A$ may believe that the probability of " $B$ has received my email" is less than 0.99 , or may believe that the probability of " $B$ has received my email" is at least 0.8 , and so on. On the other hand, $B$ may believe that the probability of " $A$ believes that the probability of ' $B$ has received my email' is at least 0.9 " is less than 0.8. In order to reply to $A, B$ sends an acknowledgement email to $A, A$ receives the email, and sends another acknowledgement email to $B$, now $B$ believes that the probability of " $A$ believes that the probability of ' $B$ has received my first email' is equal to 1 " is equal to 1 . In order to represent and reason with probabilistic belief, it is necessary to extend belief logic to probabilistic belief logic. In following, we propose a probabilistic belief logic $P B L_{\omega}$, the basic formula in $P B L_{\omega}$ is $B_{i}(a, \varphi)$, which says agent $i$ believes that the probability of $\varphi$ is no less than $a$.

\subsection{Language of $P B L_{\omega}$}

Throughout this chapter, we let $L^{P B L_{\omega}}$ be a language which is just the set of formulas of interest to us.

Definition 2.1 The set of formulas in $P B L_{\omega}$, called $L^{P B L_{\omega}}$, is given by the following rules:

(1) If $\varphi \in$ Atomic formulas set Prop, then $\varphi \in L^{P B L_{\omega}}$;

(2) If $\varphi \in L^{P B L_{\omega}}$, then $\neg \varphi \in L^{P B L_{\omega}}$;

(3) If $\varphi_{1}, \varphi_{2} \in L^{P B L_{\omega}}$, then $\varphi_{1} \wedge \varphi_{2} \in L^{P B L_{\omega}}$;

(4) If $\varphi \in L^{P B L_{\omega}}$ and $a \in[0,1]$, then $B_{i}(a, \varphi) \in L^{P B L_{\omega}}$, where $i$ belongs to the set of agents $\{1, \ldots, n\}$. Intuitively, $B_{i}(a, \varphi)$ means that agent $i$ believes the probability of $\varphi$ is no less than $a$.

\subsection{Semantics of $P B L_{\omega}$}

We will describe the semantics of $P B L_{\omega}$, i.e., a formal model that we can use to determine whether a given formula is true or false. We call the formal model probabilistic model, roughly speaking, at each state, each agent has a probability on a certain set of states.

Definition 2.2 A probabilistic model $P M$ of $P B L_{\omega}$ is a tuple $\left(S, \pi, P_{1}, \ldots, P_{n}\right)$, where

(1) $S$ is a nonempty set, whose elements are called possible worlds or states;

(2) $\pi$ is a map: $S \times$ Prop $\rightarrow\{$ true, false $\}$, where Prop is a set of atomic formulas;

(3) $P_{i}$ is a map, it maps every possible world $s$ to a $P B L_{\omega}$-probability space $P_{i}(s)=$ $\left(S, X_{i, s}, \mu_{i, s}\right)$.

Where $X_{i, s} \in \wp(S)$, which satisfies the following conditions: 
(a) If $p$ is an atomic formula, then $e v_{P M}(p)=\left\{s^{\prime} \mid \pi\left(s^{\prime}, p\right)=\right.$ true $\} \in X_{i, s}$;

(b) If $A \in X_{i, s}$, then $S-A \in X_{i, s}$;

(c) If $A_{1}, A_{2} \in X_{i, s}$, then $A_{1} \cap A_{2} \in X_{i, s}$;

(d) If $A \in X_{i, s}$ and $a \in[0,1]$, then $\left\{s^{\prime} \mid \mu_{i, s^{\prime}}(A) \geq a\right\} \in X_{i, s}$.

$\mu_{i, s}$ is a $P B L_{\omega}$ - finite additivity probability measure assigned to the set $X_{i, s}$, i.e., $\mu_{i, s}$ satisfies the following conditions:

(a) $\mu_{i, s}(A) \geq 0$ for all $A \in X_{i, s}$;

(b) $\mu_{i, s}(S)=1$;

(c) (finite additivity) $\mu_{i, s}\left(A_{1} \cup A_{2}\right)=\mu_{i, s}\left(A_{1}\right)+\mu_{i, s}\left(A_{2}\right)$, where $A_{1}$ and $A_{2}$ are disjoint members of $X_{i, s}$;

(d) If $A \in X_{i, s}$ and $\mu_{i, s}(A) \geq a$, then $\mu_{i, s}\left(\left\{s^{\prime} \mid \mu_{i, s^{\prime}}(A) \geq a\right\}\right)=1$; if $A \in X_{i, s}$ and $\mu_{i, s}(A)<b$, then $\mu_{i, s}\left(\left\{s^{\prime} \mid \mu_{i, s^{\prime}}(A)<b\right\}\right)=1$.

Notice that from the definition of $X_{i, s}$, we have $\left\{s^{\prime} \mid \mu_{i, s^{\prime}}(A) \geq a\right\} \in X_{i, s}$ and $\left\{s^{\prime} \mid \mu_{i, s^{\prime}}(A)<\right.$ $b\}=S-\left\{s^{\prime} \mid \mu_{i, s^{\prime}}(A) \geq b\right\} \in X_{i, s}$. Intuitively, the probability space $P_{i}(s)$ describes agent $i^{\prime} s$ probabilities on events, given that the state is $s$. $W$ is the sample space, which is the set of states that agent $i$ considers possible. $X_{i, s}$ is the set of measurable sets. The measure $\mu_{i, s}$ does not assign a probability to all subsets of $S$ but only to the measurable sets.

As an example, we consider a $P B L_{\omega}$-model such that $P M=\left(S, \pi, P_{1}\right)$. Here $S=\left\{s_{1}, s_{2}, s_{3}\right\}$; $\pi\left(s_{1}, p\right)=$ false, $\pi\left(s_{2}, p\right)=$ false,$\pi\left(s_{3}, p\right)=$ true, $\pi\left(s_{1}, q\right)=$ true, $\pi\left(s_{2}, q\right)=$ true, $\pi\left(s_{3}, q\right)=$ true; $P_{1}$ is defined as follows: for every $s \in S, P_{1}(s)=\left(S, X_{1, s}, \mu_{1, s}\right)$, where $X_{1, s_{1}}=X_{1, s_{2}}=\wp(S), X_{1, s_{3}}=\left\{\varnothing,\left\{s_{1}, s_{2}\right\},\left\{s_{3}\right\}, S\right\}, \mu_{1, s_{1}}(\varnothing)=\mu_{1, s_{2}}(\varnothing)=\mu_{1, s_{3}}(\varnothing)=0$, $\mu_{1, s_{1}}\left(\left\{s_{1}\right\}\right)=\mu_{1, s_{2}}\left(\left\{s_{1}\right\}\right)=1 / 2, \mu_{1, s_{1}}\left(\left\{s_{2}\right\}\right)=\mu_{1, s_{2}}\left(\left\{s_{2}\right\}\right)=1 / 2, \mu_{1, s_{1}}\left(\left\{s_{3}\right\}\right)=\mu_{1, s_{2}}\left(\left\{s_{3}\right\}\right)=$ $0, \mu_{1, s_{3}}\left(\left\{s_{3}\right\}\right)=1, \mu_{1, s_{1}}\left(\left\{s_{1}, s_{2}\right\}\right)=\mu_{1, s_{2}}\left(\left\{s_{1}, s_{2}\right\}\right)=1, \mu_{1, s_{3}}\left(\left\{s_{1}, s_{2}\right\}\right)=0, \mu_{1, s_{1}}\left(\left\{s_{1}, s_{3}\right\}\right)=$ $\mu_{1, s_{2}}\left(\left\{s_{1}, s_{3}\right\}\right)=1 / 2, \mu_{1, s_{1}}\left(\left\{s_{2}, s_{3}\right\}\right)=\mu_{1, s_{2}}\left(\left\{s_{2}, s_{3}\right\}\right)=1 / 2, \mu_{1, s_{1}}(S)=\mu_{1, s_{2}}(S)=\mu_{1, s_{3}}(S)=$ 1. It is easy to check that the above model satisfies the conditions in Definition 2.2. In this model, it is clear that the set of measurable sets $X_{1, s}$ and probability measure $\mu_{1, s}$ varies with $s$, and consequently the probability space also varies with $s$, hence we index probability space by $s$.

We now define what it means for a formula to be true at a given world $s$ in a probabilistic model $P M$.

Definition 2.3 Probabilistic semantics of $P B L_{\omega}$

$(P M, s) \models p$ iff $\pi(s, p)=$ true, where $p$ is an atomic formula;

$(P M, s) \models \neg \varphi$ iff $(P M, s) \not \models \varphi$;

$(P M, s) \models \varphi \wedge \psi$ iff $(P M, s) \models \varphi$ and $(P M, s) \models \psi$;

$(P M, s) \models B_{i}(a, \varphi)$ iff $\mu_{i, s}\left(e v_{P M}(\varphi)\right) \geq a$, where $e v_{P M}(\varphi)=\left\{s^{\prime} \mid\left(P M, s^{\prime}\right) \models \varphi\right\}$.

The intuitive meaning of the semantics of $B_{i}(a, \varphi)$ is that agent $i$ believes that the probability of $\varphi$ is at least $a$ in world $(P M, s)$ if the measure of possible worlds satisfying $\varphi$ is at least $a$. 
In the above example, according to Definition 2.3, we have $\left(P M, s_{1}\right) \models B_{1}(1 / 2, q),\left(P M, s_{2}\right) \models$ $B_{1}(0, p \wedge q),\left(P M, s_{3}\right) \models B_{1}(1, p \wedge q)$, etc.

In order to characterize the properties of probabilistic belief, we will characterize the formulas that are always true. More formally, given a probabilistic model $P M$, we say that $\varphi$ is valid in $P M$, and write $P M \models \varphi$, if $(P M, s) \models \varphi$ for every state $s$ in $S$, and we say that $\varphi$ is satisfiable in $P M$ if $(P M, s) \models \varphi$ for some $s$ in $S$. We say that $\varphi$ is valid, and write $\models \varphi$, if $\varphi$ is valid in all probabilistic models, and that $\varphi$ is satisfiable if it is satisfiable in some probabilistic model. We write $\Gamma \models \varphi$, if $\varphi$ is valid in all probabilistic models in which $\Gamma$ is satisfiable.

\subsection{Inference system of $P B L_{\omega}$}

Now we list a number of valid properties of probabilistic belief, which form the inference system of $P B L_{\omega}$.

\section{Axioms and inference rules of proposition logic}

Axiom $1 . B_{i}(0, \varphi)$ (For any proposition $\varphi$, agent $i$ believes that the probability of $\varphi$ is no less than 0.$)$

Axiom 2. $B_{i}(a, \varphi) \wedge B_{i}(b, \psi) \rightarrow B_{i}(\max (a+b-1,0), \varphi \wedge \psi)$ (For any $\varphi$ and $\psi$, if agent $i$ believes that the probability of $\varphi$ is no less than $a$, and believes that the probability of $\psi$ is no less than $b$, then agent $i$ believes that the probability of $\varphi \wedge \psi$ is no less than $\max (a+b-1,0)$.)

Axiom 3. $B_{i}(a, \varphi) \rightarrow B_{i}\left(1, B_{i}(a, \varphi)\right)$ (If agent $i$ believes that the probability of $\varphi$ is no less than $a$, then agent $i$ believes that the probability of his belief being true is no less than 1.)

Axiom 4. $\neg B_{i}(a, \varphi) \rightarrow B_{i}\left(1, \neg B_{i}(a, \varphi)\right)$ (If agent $i$ believes that the probability of $\varphi$ is less than $a$, then agent $i$ believes that the probability of his belief being true is no less than 1.)

Axiom 5. $B_{i}(a, \varphi) \rightarrow B_{i}(b, \varphi)$, where $1 \geq a \geq b \geq 0$. (If agent $i$ believes that the probability of $\varphi$ is no less than $a$, and $1 \geq a \geq b \geq 0$, then agent $i$ believes that the probability of $\varphi$ is no less than $b$.)

Axiom 6. $B_{i}(a+b, \varphi \vee \psi) \rightarrow\left(B_{i}(a, \varphi) \vee B_{i}(b, \psi)\right)$, where $1 \geq a+b \geq 0$. (If agent $i$ believes that the probability of $\varphi \vee \psi$ is no less than $a+b$, then agent $i$ believes that the probability of $\varphi$ is no less than $a$ or believes that the probability of $\psi$ is no less than $b$.)

Rule 1. $\vdash \varphi \Rightarrow \vdash B_{i}(1, \varphi)$ (If $\varphi$ is a tautology proposition, then agent $i$ believes that the probability of $\varphi$ is no less than 1.)

Rule 2. $\vdash \varphi \rightarrow \psi \Rightarrow \vdash B_{i}(a, \varphi) \rightarrow B_{i}(a, \psi)$ (If $\varphi \rightarrow \psi$ is a tautology proposition, and agent $i$ believes that the probability of $\varphi$ is no less than $a$, then agent $i$ believes that the probability of $\psi$ is no less than $a$.)

Rule 3. $\vdash \neg(\varphi \wedge \psi) \Rightarrow \vdash \neg\left(B_{i}(a, \varphi) \wedge B_{i}(b, \psi)\right)$ for any $a, b \in[0,1]$ such that $a+b>1$. (If $\varphi$ and $\psi$ are incompatible propositions, then it is impossible that agent $i$ believes that the probability of $\varphi$ is no less than $a$, and believes that the probability of $\psi$ is no less than $b$, where $a+b>1$.)

Rule 4. $\vdash \neg(\varphi \wedge \psi) \Rightarrow \vdash B_{i}(a, \varphi) \wedge B_{i}(b, \psi) \rightarrow B_{i}(a+b, \varphi \vee \psi)$, where $a+b \leq 1$. (If $\varphi$ and $\psi$ are incompatible propositions, agent $i$ believes that the probability of $\varphi$ is no less than $a$, and believes that the probability of $\psi$ is no less than $b$, where $a+b \leq 1$, then agent $i$ believes that the probability of $\varphi \vee \psi$ is no less than $a+b$.) 
Rule 5. $\Gamma \vdash B_{i}\left(a_{n}, \varphi\right)$ for all $n \in M \Rightarrow \Gamma \vdash B_{i}(a, \varphi)$, where $a=\sup _{n \in M}\left(\left\{a_{n}\right\}\right)$. (If agent $i$ believes that the probability of $\varphi$ is no less than $a_{n}$, where $n$ is any element in the index set $M$, then agent $i$ believes that the probability of $\varphi$ is no less than $a$, where $a=\sup _{n \in M}\left(\left\{a_{n}\right\}\right)$.)

Rule 6. Given a set of formulas $\Sigma, \Gamma \cup\left(\cup_{\varphi \in \Sigma}\left(\left\{B_{i}(a, \varphi) \mid 0 \leq a \leq a_{i, \varphi}\right\} \cup\left\{\neg B_{i}(b, \varphi) \mid 1 \geq b>\right.\right.\right.$ $\left.\left.\left.a_{i, \varphi}\right\}\right)\right) \vdash \psi$ for any $a_{i, \varphi} \in[0,1] \Rightarrow \Gamma \vdash \psi$. (If $\psi$ can be proved from $\Gamma$ with any possible probabilistic belief of agent $i$ for $\Sigma$, then $\psi$ can be merely proved from $\Gamma$.)

Remark: In Rule 5, the index set $M$ may be an infinite set, therefore we call Rule 5 an infinite inference rule. For example, let $\Gamma=\left\{B_{i}(1 / 2, \varphi), B_{i}(2 / 3, \varphi), \ldots, B_{i}(n / n+1, \varphi), \ldots\right\}$, we have $\Gamma \vdash B_{i}(n / n+1, \varphi)$ for all $n \in M=\{1,2, \ldots, k, \ldots\}$, by Rule 5 , we get $\Gamma \vdash B_{i}(1, \varphi)$ since $1=\sup _{n \in M}(\{n / n+1\})$.

In Rule 6, $\left\{B_{i}(a, \varphi) \mid 0 \leq a \leq a_{i, \varphi}\right\} \cup\left\{\neg B_{i}(b, \varphi) \mid 1 \geq b>a_{i, \varphi}\right\}$ means that agent $i$ believes the probability of $\varphi$ is exactly $a_{i, \varphi}$. Therefore $\Gamma \cup\left\{B_{i}(a, \varphi) \mid 0 \leq a \leq a_{i, \varphi}\right\} \cup\left\{\neg B_{i}(b, \varphi) \mid 1 \geq b>\right.$ $\left.a_{i, \varphi}\right\} \vdash \psi$ for any $a_{i, \varphi} \in[0,1]$ means that under any possible probabilistic belief of agent $i$ for $\varphi, \psi$ can be proved from $\Gamma$. Intuitively, in this case, the correctness of $\psi$ is independent of the exact probability of $\varphi$ that agent $i$ believes, so we can get $\psi$ from $\Gamma$. In Rule 6 , formula $\varphi$ here is generalized to arbitrary set $\Sigma$ of formulas. Since the premises of Rule 6 are infinite, it is also an infinite inference rule.

We will show that in a precise sense these properties completely characterize the formulas of $P B L_{\omega}$ that are valid with respect to probabilistic model. To do so, we have to consider the notion of provability. Inference system $P B L_{\omega}$ consists of a collection of axioms and inference rules. We are actually interested in (substitution) instances of axioms and inference rules (so we in fact think of axioms and inference rules as schemes). For example, the formula $B_{i}(0.7, \varphi) \wedge B_{i}(0.8, \psi) \rightarrow B_{i}(0.5, \varphi \wedge \psi)$ is an instances of the propositional tautology $B_{i}(a, \varphi) \wedge B_{i}(b, \psi) \rightarrow B_{i}(\max (a+b-1,0), \varphi \wedge \psi)$, obtained by substituting $B_{i}(0.7, \varphi), B_{i}(0.8, \psi)$ and $B_{i}(0.5, \varphi \wedge \psi)$ for $B_{i}(a, \varphi), B_{i}(b, \psi)$ and $B_{i}(\max (a+b-1,0), \varphi \wedge \psi)$ respectively. A proof in $P B L_{\omega}$ consists of a sequence of formulas, each of which is either an instance of an axiom in $P B L_{\omega}$ or follows from an application of an inference rule. (If " $\varphi_{1}, \ldots, \varphi_{n}$ infer $\psi$ " is an instance of an inference rule, and if the formulas $\varphi_{1}, \ldots, \varphi_{n}$ have appeared earlier in the proof, then we say that $\psi$ follows from an application of an inference rule.) A proof is said to be from $\Gamma$ to $\varphi$ if the premise is $\Gamma$ and the last formula is $\varphi$ in the proof. We say $\varphi$ is provable from $\Gamma$ in $P B L_{\omega}$, and write $\Gamma \vdash_{P B L_{\omega}} \varphi$, if there is a proof from $\Gamma$ to $\varphi$ in $P B L_{\omega}$.

\subsection{Soundness of $P B L_{\omega}$}

We will prove that $P B L_{\omega}$ characterizes the set of formulas that are valid with respect to probabilistic model. Inference system of $P B L_{\omega}$ is said to be sound with respect to probabilistic models if every formula provable in $P B L_{\omega}$ is valid with respect to probabilistic models. The system $P B L_{\omega}$ is complete with respect to probabilistic models if every formula valid with respect to probabilistic models is provable in $P B L_{\omega}$. We think of $P B L_{\omega}$ as characterizing probabilistic models if it provides a sound and complete axiomatization of that class; notationally, this amounts to saying that for all formulas set $\Gamma$ and all formula $\varphi$, we have $\Gamma \vdash_{P B L_{\omega}} \varphi$ if and only if $\Gamma \models_{P B L_{\omega}} \varphi$. The following soundness and completeness provide a tight connection between the syntactic notion of provability and the semantic notion of validity.

Firstly, we need the following obvious lemmas. 
Lemma 2.1 $\mu_{i, s}\left(A_{1} \cup A_{2}\right)=\mu_{i, s}\left(A_{1}\right)+\mu_{i, s}\left(A_{2}\right)$, here $A_{1}$ and $A_{2}$ are any disjoint members of $X_{i, s} \Rightarrow$ for any members $A_{1}$ and $A_{2}$ of $X_{i, s}, \mu_{i, s}\left(A_{1} \cup A_{2}\right)+\mu_{i, s}\left(A_{1} \cap A_{2}\right)=\mu_{i, s}\left(A_{1}\right)+\mu_{i, s}\left(A_{2}\right)$.

Proof. $\mu_{i, s}\left(A_{1} \cup A_{2}\right)+\mu_{i, s}\left(A_{1} \cap A_{2}\right)=\mu_{i, s}\left(A_{1} \cup\left(A_{2}-A_{1}\right)\right)+\mu_{i, s}\left(A_{1} \cap A_{2}\right)=\mu_{i, s}\left(A_{1}\right)+$ $\mu_{i, \mathrm{~s}}\left(A_{1} \cap A_{2}\right)+\mu_{i, s}\left(A_{2}-A_{1}\right)=\mu_{i, s}\left(A_{1}\right)+\mu_{i, s}\left(\left(A_{1} \cap A_{2}\right) \cup\left(A_{2}-A_{1}\right)\right)=\mu_{i, s}\left(A_{1}\right)+\mu_{i, s}\left(A_{2}\right)$.

Lemma $2.2 \mu_{i, s}\left(A_{1} \cap A_{2}\right) \geq \mu_{i, s}\left(A_{1}\right)+\mu_{i, s}\left(A_{2}\right)-1$.

Proof. It follows from Lemma 2.1 immediately.

Lemma $2.3 \mu_{i, s}\left(A_{1}\right)+\mu_{i, S}\left(A_{2}\right) \geq \mu_{i, s}\left(A_{1} \cup A_{2}\right)$. If $A_{1} \cap A_{2}=\varnothing$, then $\mu_{i, s}\left(A_{1}\right)+\mu_{i, S}\left(A_{2}\right)=$ $\mu_{i, s}\left(A_{1} \cup A_{2}\right)$.

Proof. It follows from Lemma 2.1 .

Now, we can prove the following proposition:

Proposition 2.1 (Soundness of $P B L_{\omega}$ ) If $\Gamma \vdash_{P B L_{\omega}} \varphi$, then $\Gamma \models_{P B L_{\omega}} \varphi$.

Proof. We show each axiom and each rule of $P B L_{\omega}$ is sound, respectively.

Axiom 1: By the definition of $P B L_{\omega}$-probability measure, for any s, if $A \in X_{i, s}$ then $\mu_{i, s}(A) \geq 0$. Since by the definition of $X_{i, s}$, for any $\varphi, e v_{P M}(\varphi)=\left\{s^{\prime} \mid\left(P M, s^{\prime}\right) \models \varphi\right\} \in X_{i, s}$, we have $\mu_{i, s}\left(e v_{P M}(\varphi)\right) \geq 0$, therefore $B_{i}(0, \varphi)$ holds.

Axiom 2: Suppose $(P M, s) \models B_{i}(a, \varphi) \wedge B_{i}(b, \psi)$, so $\mu_{i, s}\left(e v_{P M}(\varphi)\right) \geq a$ and $\mu_{i, s}\left(e v_{P M}(\psi)\right) \geq$ $b$. For $\mu_{i, s}$ is $P B L_{\omega}$ - probability measure, by Lemma 2.2, we get $\mu_{i, s}\left(e v_{P M}(\varphi \wedge \psi)\right)=$ $\mu_{i, s}\left(e v_{P M}(\varphi) \cap e v_{P M}(\psi)\right) \geq \mu_{i, s}\left(e v_{P M}(\varphi)\right)+\mu_{i, s}\left(e v_{P M}(\psi)\right)-1 \geq a+b-1$, which implies $(P M, s) \models B_{i}(\max (a+b-1,0), \varphi \wedge \psi)$.

Axiom 3: Suppose $(P M, s) \models B_{i}(a, \varphi)$, so $\mu_{i, s}\left(e v_{P M}(\varphi)\right) \geq a$. Let $\Lambda_{i}^{a}(\varphi)=$ $\left\{s^{\prime} \mid \mu_{i, s^{\prime}}\left(e v_{P M}(\varphi)\right) \geq a\right\}$, by the definition of $\mu_{i, s}$, we get $\mu_{i, s}\left(\Lambda_{i}^{a}(\varphi)\right)=1$. Assume $s^{\prime} \in \Lambda_{i}^{a}(\varphi)$, then $s^{\prime} \in e v_{P M}\left(B_{i}(a, \varphi)\right)$, hence $\Lambda_{i}^{a}(\varphi) \subseteq e v_{P M}\left(B_{i}(a, \varphi)\right)$, so $\mu_{i, s}\left(e v_{P M}\left(B_{i}(a, \varphi)\right)\right)=1$, which implies $(P M, s) \models B_{i}\left(1, B_{i}(a, \varphi)\right)$.

Axiom 4: Suppose $(P M, s) \models \neg B_{i}(a, \varphi)$, so $\mu_{i, s}\left(\operatorname{ev}_{P M}(\varphi)\right)<a$. Let $\Theta_{i}^{a}(\varphi)=$ $\left\{s^{\prime} \mid \mu_{i, s^{\prime}}\left(e v_{P M}(\varphi)\right)<a\right\}$, by the definition of $\mu_{i, s}$, we get $\mu_{i, s}\left(\Theta_{i}^{a}(\varphi)\right)=1$. Assume $s^{\prime} \in \Theta_{i}^{a}(\varphi)$, then $s^{\prime} \in e v_{P M}\left(\neg B_{i}(a, \varphi)\right)$, hence $\mu_{i, s}\left(e v_{P M}\left(\neg B_{i}(a, \varphi)\right)\right)=1$, which implies $(P M, s) \vDash$ $B_{i}\left(1, \neg B_{i}(a, \varphi)\right)$.

Axiom 5: Suppose $(P M, s) \models B_{i}(a, \varphi)$, so $\mu_{i, s}\left(e v_{P M}(\varphi)\right) \geq a$. If $1 \geq a \geq b \geq 0$, then $\mu_{i, s}\left(\operatorname{ev}_{P M}(\varphi)\right) \geq b$, so $(P M, s) \models B_{i}(b, \varphi)$, therefore $B_{i}(a, \varphi) \rightarrow B_{i}(b, \varphi)$ holds.

Axiom 6: Suppose $(P M, s) \models B_{i}(a+b, \varphi \vee \psi)$, then $\mu_{i, s}\left(e v_{P M}(\varphi \vee \psi)\right) \geq a+b$. Since $\mu_{i, s}\left(e v_{P M}(\varphi)\right)+\mu_{i, s}\left(e v_{P M}(\psi)\right) \geq \mu_{i, s}\left(e v_{P M}(\varphi) \cup e v_{P M}(\psi)\right) \geq a+b$, we have $\mu_{i, s}\left(e v_{P M}(\varphi)\right) \geq$ $a$ or $\mu_{i, s}\left(e v_{P M}(\psi)\right) \geq b$. Hence $(P M, s) \models B_{i}(a, \varphi) \vee B_{i}(b, \psi)$.

Rule 1: Since $\models \varphi$, so for any possible world $s, \mu_{i, s}\left(\operatorname{ev}_{P M}(\varphi)\right) \geq 1$, therefore $\models B_{i}(1, \varphi)$ holds.

Rule 2: Since $\models \varphi \rightarrow \psi$, so $e v_{P M}(\varphi) \subseteq e v_{P M}(\psi)$. Suppose $(P M, s) \models B_{i}(a, \varphi)$, therefore $\mu_{i, s}\left(e v_{P M}(\varphi)\right) \geq a$, by the property of $P B L_{\omega}$-probability space, we get $\mu_{i, s}\left(e v_{P M}(\varphi)\right) \leq$ $\mu_{i, s}\left(e v_{P M}(\psi)\right)$. So $\mu_{i, s}\left(e v_{P M}(\psi)\right) \geq a$. Therefore $(P M, s) \mid=B_{i}(a, \psi)$, and Rule 2 of $P B L_{\omega}$ holds.

Rule 3: Suppose $\models \neg(\varphi \wedge \psi)$, so $e v_{P M}(\varphi) \cap e v_{P M}(\psi)=\varnothing$. By the property of $P B L_{\omega}$-probability space and Lemma 2.3 , for any possible world $s$, we get $\mu_{i, s}\left(e v_{P M}(\varphi) \cup\right.$ 
$\left.e v_{P M}(\psi)\right)=\mu_{i, s}\left(e v_{P M}(\varphi)\right)+\mu_{i, s}\left(e v_{P M}(\psi)\right)$ and $\mu_{i, s}\left(e v_{P M}(\varphi) \cup e v_{P M}(\psi)\right) \leq 1$, therefore $\mu_{i, s}\left(e v_{P M}(\varphi)\right)+\mu_{i, s}\left(e v_{P M}(\psi)\right) \leq 1$. Assume $(P M, s) \models\left(B_{i}(a, \varphi) \wedge B_{i}(b, \psi)\right)$ where $a+b>1$, then $\mu_{i, s}\left(e v_{P M}(\varphi)\right) \geq a, \mu_{i, s}\left(e v_{P M}(\psi)\right) \geq b$, but $a+b>1$, it is a contradiction.

Rule 4: Suppose $\models \neg(\varphi \wedge \psi)$ and for possible world $s,(P M, s) \models B_{i}(a, \varphi) \wedge B_{i}(b, \psi)$, so $e v_{P M}(\varphi) \cap e v_{P M}(\psi)=\varnothing, \mu_{i, s}\left(e v_{P M}(\varphi)\right) \geq a$, and $\mu_{i, s}\left(e v_{P M}(\psi)\right) \geq b$. By the property of $P B L_{\omega}$-probability space and Lemma 2.3 , for any possible world $s$, we get $\mu_{i, s}\left(e v_{P M}(\varphi) \cup\right.$ $\left.e v_{P M}(\psi)\right)=\mu_{i, s}\left(e v_{P M}(\varphi)\right)+\mu_{i, s}\left(e v_{P M}(\psi)\right)$. Hence, $\mu_{i, s}\left(e v_{P M}(\varphi)\right)+\mu_{i, s}\left(e v_{P M}(\psi)\right) \geq a+b$ and $\mu_{i, s}\left(e v_{P M}(\varphi) \cup e v_{P M}(\psi)\right) \geq a+b$, which means $(P M, s) \models B_{i}(a+b, \varphi \vee \psi)$.

Rule 5: Suppose $\Gamma \models B_{i}\left(a_{n}, \varphi\right)$ for all $n \in M$, therefore for every $s$, if $(P M, s) \models \Gamma$, then $(P M, s)=B_{i}\left(a_{n}, \varphi\right)$ for all $n \in M$, so $\mu_{i, s}\left(e v_{P M}(\varphi)\right) \geq a_{n}$ for all $n \in M$. We get $\mu_{i, s}\left(\operatorname{ev}_{P M}(\varphi)\right) \geq \sup _{n \in M}\left(\left\{a_{n}\right\}\right)$. Therefore, $(P M, s) \models B_{i}(a, \varphi)$ and $a=\sup _{n \in M}\left(\left\{a_{n}\right\}\right)$, we get $\Gamma \models B_{i}(a, \varphi)$ and $a=\sup _{n \in M}\left(\left\{a_{n}\right\}\right)$ as desired.

Rule 6: Suppose $\Gamma \cup\left(\cup_{\varphi \in \Sigma}\left(\left\{B_{i}(a, \varphi) \mid 0 \leq a \leq a_{i, \varphi}\right\} \cup\left\{\neg B_{i}(b, \varphi) \mid 1 \geq b>a_{i, \varphi}\right\}\right)\right) \models \psi$ for any $a_{i, \varphi} \in[0,1]$, let $(P M, s) \models \Gamma$ and $c_{i, \varphi}=\mu_{i, s}\left(e v_{P M}(\varphi)\right)$, it is clear that $c_{i, \varphi} \in[0,1]$ and $(P M, s) \models$ $\Gamma \cup\left(\cup_{\varphi \in \Sigma}\left(\left\{B_{i}(a, \varphi) \mid 0 \leq a \leq c_{i, \varphi}\right\} \cup\left\{\neg B_{i}(b, \varphi) \mid 1 \geq b>c_{i, \varphi}\right)\right)\right.$. Since $\Gamma \cup\left(\cup_{\varphi \in \Sigma}\left(\left\{B_{i}(a, \varphi) \mid 0 \leq\right.\right.\right.$ $\left.\left.\left.a \leq a_{i, \varphi}\right\} \cup\left\{\neg B_{i}(b, \varphi) \mid 1 \geq b>a_{i, \varphi}\right\}\right)\right) \models \psi$ for any $a_{i, \varphi} \in[0,1]$, we have $(P M, s) \models \psi$, therefore $\Gamma \models \psi$.

\subsection{Completeness of $P B L_{\omega}$}

We shall show that the inference system of $P B L_{\omega}$ provides a complete axiomatization for probabilistic belief with respect to a probabilistic model. To achieve this aim, it suffices to prove that every $P B L_{\omega}$-consistent set is satisfiable with respect to a probabilistic model. We prove this by using a general technique that works for a wide variety of probabilistic modal logic. We construct a special structure $P M$ called a canonical structure for $P B L_{\omega}$. $P M$ has a state $s_{V}$ corresponding to every maximal $P B L_{\omega}$-consistent set $V$ and the following property holds: $\left(P M, s_{V}\right) \models \varphi$ iff $\varphi \in V$.

We need some definitions before giving the proof of the completeness. Given an inference system of $P B L_{\omega}$, we say a set of formulas $\Gamma$ is a consistent set with respect to $L^{P B L_{\omega}}$ exactly if false is not provable from $\Gamma$. A set of formulas $\Gamma$ is a maximal consistent set with respect to $L^{P B L_{\omega}}$ if (1) it is $P B L_{\omega}$-consistent, and (2) for all $\varphi$ in $L^{P B L_{\omega}}$ but not in $\Gamma$, the set $\Gamma \cup\{\varphi\}$ is not $P B L_{\omega}$-consistent.

Definition 2.7 The probabilistic model $P M$ with respect to $P B L_{\omega}$ is $\left(S, P_{1}, \ldots, P_{n}, \pi\right)$.

(1) $S=\left\{\Gamma \mid \Gamma\right.$ is a maximal consistent set with respect to $\left.P B L_{\omega}\right\}$;

(2) $P_{i}$ maps every element of $S$ to a probability space: $P_{i}(\Gamma)=\left(S, X_{i, \Gamma}, \mu_{i, \Gamma}\right)$, where $X_{i, \Gamma}=$ $\left\{X(\varphi) \mid \varphi\right.$ is a formula of $\left.P B L_{\omega}\right\}$, here $X(\varphi)=\left\{\Gamma^{\prime} \mid \varphi \in \Gamma^{\prime}\right\} ; \mu_{i, \Gamma}$ is a probability assignment: $X_{i, \Gamma} \rightarrow[0,1]$, and $\mu_{i, \Gamma}(X(\varphi))=\sup \left(\left\{a \mid B_{i}(a, \varphi) \in \Gamma\right\}\right)$;

(3) $\pi$ is a truth assignment as follows: for any atomic formula $p, \pi(p, \Gamma)=$ true $\Leftrightarrow p \in \Gamma$.

Lemma 2.4 $S$ is a nonempty set.

Proof. Since the rules and axioms of $P B L_{\omega}$ are consistent, $S$ is nonempty.

Lemma 2.5 $X_{i, \Gamma}$ satisfies the conditions of Definition 2.2. 
Proof. We only prove the following claim: if $A \in X_{i, \Gamma}$, then $\left\{\Gamma^{\prime} \mid \mu_{i, \Gamma^{\prime}}(A) \geq a\right\} \in X_{i, \Gamma}$. Other cases can be proved similarly. Since $A \in X_{i, \Gamma}$, so there is $\varphi$ with $A=X(\varphi)$. It is clear that $X\left(B_{i}(a, \varphi)\right) \in X_{i, \Gamma}$, so $\left\{\Gamma^{\prime} \mid \mu_{i, \Gamma^{\prime}}(A) \geq a\right\}=\left\{\Gamma^{\prime} \mid \mu_{i, \Gamma^{\prime}}(X(\varphi)) \geq a\right\}=X\left(B_{i}(a, \varphi)\right) \in X_{i, \Gamma}$.

In classical logic, it is easy to see that every consistent set of formulas can be extended to a maximal consistent set, but with the infinitary rules in $P B L_{\omega}$ it cannot simply be proved in a naive fashion because the union of an increasing sequence of consistent sets need no longer be consistent. Therefore we give a detailed proof for this claim in the following lemma.

Lemma 2.6 For any $P B L_{\omega}$-consistent set of formulas $\triangle$, there is a maximal $P B L_{\omega}$-consistent set $\Gamma$ such that $\Delta \subseteq \Gamma$.

Proof. To show that $\Delta$ can be extended to a maximal $P B L_{\omega}$-consistent set, we construct a sequence $\Gamma_{0}, \Gamma_{1}, \ldots$ of $P B L_{\omega}$-consistent sets as follows. Let $\psi_{1}, \psi_{2}, \ldots$ be a sequence of the formulas in $L^{P B L_{\omega}}$. This sequence is not an enumeration sequence since the cardinal number of the set of real number is not enumerable, however, we can get a well-ordered sequence of the formulas by the choice axiom of set theory.

At first, we construct $\Gamma_{0}$ which satisfies the following conditions:

(1) $\Delta \subseteq \Gamma_{0}$;

(2) $\Gamma_{0}$ is consistent;

(3) For any agent $i \in\{1, \ldots, n\}$ and every $\varphi \in L^{P B L_{\omega}}$, there is some $c_{i, \varphi} \in[0,1]$ such that $\left\{B_{i}(a, \varphi) \mid 0 \leq a \leq c_{i, \varphi}\right\} \cup\left\{\neg B_{i}(b, \varphi) \mid 1 \geq b>c_{i, \varphi}\right\} \subseteq \Gamma_{0}$.

Let $\Sigma_{0}=\Delta$, then for agent 1 and every $\varphi \in L^{P B L_{\omega}}$, there is some $c_{1, \varphi} \in[0,1]$ such that $\Sigma_{0} \cup\left(\cup_{\varphi \in L^{P B L_{\omega}}}\left(\left\{B_{1}(a, \varphi) \mid 0 \leq a \leq c_{1, \varphi}\right\} \cup\left\{\neg B_{1}(b, \varphi) \mid 1 \geq b>c_{1, \varphi}\right\}\right)\right)$ is consistent, otherwise, for all $a_{1, \varphi} \in[0,1], \Sigma_{0} \cup\left(\cup_{\varphi \in L^{P B L \omega}}\left(\left\{B_{1}(a, \varphi) \mid 0 \leq a \leq a_{1, \varphi}\right\} \cup\left\{\neg B_{1}(b, \varphi) \mid 1 \geq b>a_{1, \varphi}\right\}\right)\right) \vdash$ false. By Rule 6, we have $\Sigma_{0} \vdash$ false, and since $\Sigma_{0}=\Delta$ is consistent, it is a contradiction. Let $\Sigma_{1}=\Sigma_{0} \cup\left(\cup_{\varphi \in L^{P B L_{\omega}}}\left(\left\{B_{1}(a, \varphi) \mid 0 \leq a \leq c_{1, \varphi}\right\} \cup\left\{\neg B_{1}(b, \varphi) \mid 1 \geq b>c_{1, \varphi}\right\}\right)\right)$, similarly, for agent $i$ and for each $\varphi \in L^{P B L_{\omega}}$ there is $c_{i, \varphi} \in[0,1]$ such that $\Sigma_{i-1} \cup\left(\cup_{\varphi \in L^{P B L \omega}}\left(\left\{B_{i}(a, \varphi) \mid 0 \leq\right.\right.\right.$ $\left.\left.\left.a \leq c_{i, \varphi}\right\} \cup\left\{\neg B_{i}(b, \varphi) \mid 1 \geq b>c_{i, \varphi}\right\}\right)\right)$ is consistent. Let $\Sigma_{i}=\Sigma_{i-1} \cup\left(\cup_{\varphi \in L^{P B L \omega}}\left(\left\{B_{i}(a, \varphi) \mid 0 \leq\right.\right.\right.$ $\left.\left.\left.a \leq c_{i, \varphi}\right\} \cup\left\{\neg B_{i}(b, \varphi) \mid 1 \geq b>c_{i, \varphi}\right\}\right)\right)$ for $i \in\{1, \ldots, n\}$ and $\Gamma_{0}=\cup_{i \in\{1, \ldots, n\}} \Sigma_{i}=\Sigma_{n}$, here $\{1, \ldots, n\}$ is the set of agent. Since $\Sigma_{n}$ is consistent, $\Gamma_{0}$ is also consistent.

Now we inductively construct the rest of the sequence according to $\psi_{k}$ : (a) in the case of $k=$ $n+1$, take $\Gamma_{n+1}=\Gamma_{n} \cup\left\{\psi_{n+1}\right\}$ if the set is $P B L_{\omega}$-consistent and otherwise take $\Gamma_{n+1}=\Gamma_{n}$. (b) in the case that $k$ is a limit ordinal, take $\Gamma_{k}=\cup_{n<k} \Gamma_{n} \cup\left\{\psi_{k}\right\}$ if the set is $P B L_{\omega}$-consistent and otherwise take $\Gamma_{k}=\cup_{n<k} \Gamma_{n}$. Let $\Gamma=\cup \Gamma_{k}$. We will prove that $\Gamma$ is a maximal $P B L_{\omega}$-consistent set and $\Delta \subseteq \Gamma$.

Firstly, we prove that $\Gamma_{k}$ is consistent by induction. We have already known that $\Gamma_{0}$ is consistent. Now we prove the claim when $k>0$. In the case of (a), it is clear. In the case of (b), we only need to prove that $\cup_{n<k} \Gamma_{n}$ is consistent. Suppose $\cup_{n<k} \Gamma_{n}$ is not consistent, then there is a proof $C$ of falsity from $\cup_{n<k} \Gamma_{n}$. If this proof does not apply Rule 5 and Rule 6, then one of $\Gamma_{n}$ contains the formulas in the proof, since $\Gamma_{n}$ is consistent, then there is a contradiction. If this proof does apply Rule 5, since our construction of $\Gamma_{0}$ ensures: for some $c_{i, \varphi} \in[0,1]$, $\left\{B_{i}(a, \varphi) \mid 0 \leq a \leq c_{i, \varphi}\right\} \cup\left\{\neg B_{i}(b, \varphi) \mid 1 \geq b>c_{i, \varphi}\right\} \subseteq \Gamma_{0}$, hence if $\left\{B_{i}\left(a_{1}, \varphi\right), B_{i}\left(a_{2}, \varphi\right), \ldots\right\}$ can be deduced from $\cup_{n<k} \Gamma_{n}$, then $\left\{B_{i}\left(a_{1}, \varphi\right), B_{i}\left(a_{2}, \varphi\right), \ldots\right\} \cup\left\{B_{i}(c, \varphi)\right\} \subseteq \Gamma_{0}$, here $c=$ 
$\sup \left(\left\{a_{m} \mid B_{i}\left(a_{m}, \varphi\right)\right\}\right)$, hence $\Gamma_{0} \vdash B_{i}(c, \varphi)$. This proof can be transferred to a new proof $D$ of falsity from $\cup_{n<k} \Gamma_{n}$ which does not apply Rule 5, this reduces to the case that the proof does not apply Rule 5. If this proof does apply Rule 6, since our construction of $\Gamma_{0}$ ensures: for any $\varphi \in \Sigma$ there is some $c_{i, \varphi} \in[0,1], \cup_{\varphi \in \Sigma}\left(\left\{B_{i}(a, \varphi) \mid 0 \leq a \leq c_{i, \varphi}\right\} \cup\left\{\neg B_{i}(b, \varphi) \mid 1 \geq b>c_{i, \varphi}\right\}\right) \subseteq$ $\Gamma_{0}$, hence if $\left(\cup_{n<k} \Gamma_{n}\right) \cup\left(\cup_{\varphi \in \Sigma}\left(\left\{B_{i}(a, \varphi) \mid 0 \leq a \leq a_{i, \varphi}\right\} \cup\left\{\neg B_{i}(b, \varphi) \mid 1 \geq b>a_{i, \varphi}\right\}\right)\right) \vdash \psi$ for any $a_{i, \varphi} \in[0,1]$ then $\left(\cup_{n<k} \Gamma_{n}\right)=\left(\cup_{n<k} \Gamma_{n}\right) \cup \Gamma_{0} \vdash\left(\cup_{n<k} \Gamma_{n}\right) \cup\left(\cup_{\varphi \in \Sigma}\left(\left\{B_{i}(a, \varphi) \mid 0 \leq a \leq\right.\right.\right.$ $\left.\left.\left.c_{i, \varphi}\right\} \cup\left\{\neg B_{i}(b, \varphi) \mid 1 \geq b>c_{i, \varphi}\right\}\right)\right) \vdash \psi$. This proof can be transferred to a new proof $E$ of falsity from $\cup_{n<k} \Gamma_{n}$ which does not apply Rule 6, this reduces to the case that the proof does not apply Rule 6. Therefore a proof of falsity from $\cup_{n<k} \Gamma_{n}$ can be transferred to a proof without applying Rule 5 and Rule 6 . This case has been discussed above.

The proof of that $\Gamma$ is consistent is similar to the above proof of that $\cup_{n<k} \Gamma_{n}$ is consistent.

We claim that $\Gamma$ is maximal, for suppose $\psi \in L^{P B L_{\omega}}$ and $\psi \notin \Gamma$, since $\psi$ must appear in our sequence, say as $\psi_{k}$, here we assume $k$ is a successor ordinal, the case of limit ordinal $k$ can be proved similarly. If $\Gamma_{k} \cup\left\{\psi_{k}\right\}$ were $P B L_{\omega}$-consistent, then our construction would guarantee that $\psi_{k} \in \Gamma_{k+1}$. Hence $\psi_{k} \in \Gamma$. Because $\psi_{k}=\psi \notin \Gamma$, it follows that $\Gamma_{k} \cup\{\psi\}$ is not $P B L_{\omega}$-consistent. Hence $\Gamma$ is maximal.

By the above discussion, we have a maximal $P B L_{\omega}$-consistent set $\Gamma$ such that $\Delta \subseteq \Gamma$.

Lemma 2.7 For any $\Gamma, P_{i}(\Gamma)$ is well defined, i.e., for any $S \in X_{i, \Gamma}$, the value of $\mu_{i, \Gamma}(S)$ is unique.

Proof. It suffices to prove the following claim: if $S_{1}, S_{2} \in X_{i, \Gamma}$ and $S_{1}=S_{2}$, then $\mu_{i, \Gamma}\left(S_{1}\right)=$ $\mu_{i, \Gamma}\left(S_{2}\right)$. Since $S_{1}, S_{2} \in X_{i, \Gamma}$, by the construction of $X_{i, \Gamma}$, there are $\varphi$ and $\psi$ such that $S_{1}=X(\varphi)$ and $S_{2}=X(\psi)$. Assume $S_{1}=S_{2}$, then $X(\varphi)=X(\psi)$. It is clear that $\vdash \varphi \leftrightarrow \psi$, suppose not, w.l.o.g, assume $\vdash \varphi \rightarrow \psi$ does not hold, then by Lemma 2.6, $\{\varphi, \neg \psi\}$ is consistent and there is a maximal consistent set $\Gamma^{\prime}$ such that $\{\varphi, \neg \psi\} \subseteq \Gamma^{\prime}$, this contradicts $X(\varphi)=X(\psi)$. Furthermore, by rule: $\vdash \varphi \rightarrow \psi \Rightarrow \vdash B_{i}(a, \varphi) \rightarrow B_{i}(a, \psi)$, we have $\vdash B_{i}(a, \varphi) \leftrightarrow B_{i}(a, \psi)$, so $\mu_{i, \Gamma}(X(\varphi))=\mu_{i, \Gamma}(X(\psi))$.

Lemma 2.8 Let $\operatorname{Pro}_{i, \Gamma}(\varphi)=\left\{a \mid B_{i}(a, \varphi)\right.$ is in $\left.\Gamma\right\}$, then $\sup \left(\operatorname{Pro}_{i, \Gamma}(\varphi)\right) \in \operatorname{Pro}_{i, \Gamma}(\varphi)$.

Proof. Suppose $\operatorname{Pro}_{i, \Gamma}(\varphi)=\left\{a \mid B_{i}(a, \varphi)\right.$ is in $\left.\Gamma\right\}$, therefore $\Gamma \vdash B_{i}\left(a_{n}, \varphi\right)$ for all $a_{n} \in \operatorname{Pro}_{i, \Gamma}(\varphi)$, by Rule 5 of $P B L_{\omega}, \Gamma \vdash B_{i}(a, \varphi)$, where $a=\sup _{n \in M}\left(\left\{a_{n}\right\}\right)=\sup \left(\operatorname{Pro}_{i, \Gamma}(\varphi)\right)$, so we get $\sup \left(\operatorname{Pro}_{i, \Gamma}(\varphi)\right) \in \operatorname{Pro}_{i, \Gamma}(\varphi)$ as desired.

Lemma 2.9 If $A \in X_{i, \Gamma}$, then $0 \leq \mu_{i, \Gamma}(A) \leq 1$. Furthermore, $\mu_{i, \Gamma}(\varnothing)=0, \mu_{i, \Gamma}(S)=1$.

Proof. By the construction of model, it is obvious that if $A \in X_{i, \Gamma}$ then $0 \leq \mu_{i, \Gamma}(A) \leq 1$.

By rule: $\vdash \varphi \Rightarrow \vdash B_{i}(1, \varphi)$, therefore we have $\mu_{i, \Gamma}(S)=\mu_{i, \Gamma}(X($ true $))=1$ as desired. By axiom: $B_{i}(0, \varphi)$, we get $B_{i}(0$, false $)$, so $\mu_{i, \Gamma}(\varnothing)=\mu_{i, \Gamma}(X($ false $)) \geq 0$. By rule: $\vdash \neg(\varphi \wedge \psi) \Rightarrow \vdash$ $B_{i}(a, \varphi) \wedge B_{i}(b, \psi) \rightarrow B_{i}(a+b, \varphi \vee \psi)$, where $a+b \leq 1$, we have $\mu_{i, \Gamma}(S)=\mu_{i, \Gamma}(X($ true $\vee$ false $) \geq \mu_{i, \Gamma}(X($ true $))+\mu_{i, \Gamma}(X($ false $))=\mu_{i, \Gamma}(S)+\mu_{i, \Gamma}(\varnothing)$, since $\mu_{i, \Gamma}(S)=1,1 \geq 1+$ $\mu_{i, \Gamma}(\varnothing)$ holds, therefore $\mu_{i, \Gamma}(\varnothing)=0$.

Lemma 2.10 If $A_{1}$ and $A_{2}$ are disjoint members of $X_{i, \Gamma}$, then $\mu_{i, \Gamma}\left(A_{1} \cup A_{2}\right)=\mu_{i, \Gamma}\left(A_{1}\right)+$ $\mu_{i, \Gamma}\left(A_{2}\right)$.

Proof. Suppose $A_{i}=X\left(\varphi_{i}\right)$, and $\vdash \neg\left(\varphi_{1} \wedge \varphi_{2}\right)$. By Rule $4, \vdash B_{i}\left(a_{1}, \varphi_{1}\right) \wedge B_{i}\left(a_{2}, \varphi_{2}\right) \rightarrow$ $\left.B_{i}\left(a_{1}+a_{2}, \varphi_{1} \vee \varphi_{2}\right)\right)$, therefore $\mu_{i, \Gamma}\left(X\left(\varphi_{1} \vee \varphi_{2}\right)\right) \geq \mu_{i, \Gamma}\left(X\left(\varphi_{1}\right)\right)+\mu_{i, \Gamma}\left(X\left(\varphi_{2}\right)\right)$. Since $X\left(\varphi_{1} \vee\right.$ 
$\left.\varphi_{2}\right)=A_{1} \cup A_{2}$, we have $\mu_{i, \Gamma}\left(A_{1} \cup A_{2}\right)=\mu_{i, \Gamma}\left(X\left(\varphi_{1} \vee \varphi_{2}\right)\right) \geq \mu_{i, \Gamma}\left(X\left(\varphi_{1}\right)\right)+\mu_{i, \Gamma}\left(X\left(\varphi_{2}\right)\right)=$ $\mu_{i, \Gamma}\left(A_{1}\right)+\mu_{i, \Gamma}\left(A_{2}\right)$.

Now, we prove $\mu_{i, \Gamma}\left(A_{1}\right)+\mu_{i, \Gamma}\left(A_{2}\right) \geq \mu_{i, \Gamma}\left(A_{1} \cup A_{2}\right)$. Suppose $\mu_{i, \Gamma}\left(A_{1} \cup A_{2}\right)>\mu_{i, \Gamma}\left(A_{1}\right)+$ $\mu_{i, \Gamma}\left(A_{2}\right)$. Let $\mu_{i, \Gamma}\left(A_{1}\right)=a_{1}, \mu_{i, \Gamma}\left(A_{2}\right)=a_{2}, \mu_{i, \Gamma}\left(A_{1} \cup A_{2}\right)>a_{1}+a_{2}$. Choose $e>0$ such that $2 e+a_{1}+a_{2}=\mu_{i, \Gamma}\left(A_{1} \cup A_{2}\right)$, then $\mu_{i, \Gamma}\left(A_{1}\right)<a_{1}+e, \mu_{i, \Gamma}\left(A_{2}\right)<a_{2}+e$.

Therefore we have $\Gamma \models B_{i}\left(a_{1}+a_{2}+2 e, \varphi_{1} \vee \varphi_{2}\right) \wedge \neg B_{i}\left(a_{1}+e, \varphi_{1}\right) \wedge \neg B_{i}\left(a_{2}+e, \varphi_{2}\right)$. Since $\Gamma$ is a maximal consistent set, so $\Gamma \vdash B_{i}\left(a_{1}+a_{2}+2 e, \varphi_{1} \vee \varphi_{2}\right) \wedge \neg B_{i}\left(a_{1}+e, \varphi_{1}\right) \wedge \neg B_{i}\left(a_{2}+e, \varphi_{2}\right)$, which contradicts Axiom 6 of $P B L_{\omega}$.

Lemma 2.11 For any $\varphi$, let $\Delta_{i}^{a}(\varphi)=\left\{\Gamma^{\prime} \mid \mu_{i, \Gamma^{\prime}}(X(\varphi)) \geq a\right\}$. If $\mu_{i, \Gamma}(X(\varphi)) \geq a$, then $\mu_{i, \Gamma}\left(\Delta_{i}^{a}(\varphi)\right)=1$.

Proof. It is clear that $B_{i}(a, \varphi) \in \Gamma \Rightarrow \Gamma \in \Delta_{i}^{a}(\varphi)$, therefore $X\left(B_{i}(a, \varphi)\right) \subseteq \Delta_{i}^{a}(\varphi)$. Since $B_{i}(a, \varphi) \rightarrow B_{i}\left(1, B_{i}(a, \varphi)\right)$, so $B_{i}\left(1, B_{i}(a, \varphi)\right) \in \Gamma$, thereby $\mu_{i, \Gamma}\left(X\left(B_{i}(a, \varphi)\right)\right)=$ $\sup \left(\left\{b \mid B_{i}\left(b, B_{i}(a, \varphi)\right) \in \Gamma\right\}\right)=1$. For $X\left(B_{i}(a, \varphi)\right) \subseteq \Delta_{i}^{a}(\varphi)$, we have $\mu_{i, \Gamma}\left(\Delta_{i}^{a}(\varphi)\right)=1$ as desired.

Lemma 2.12 For any $\varphi$, let $\Theta_{i}^{a}(\varphi)=\left\{\Gamma^{\prime} \mid \mu_{i, \Gamma^{\prime}}(X(\varphi))<a\right\}$. If $\mu_{i, \Gamma}(X(\varphi))<a$, then $\mu_{i, \Gamma}\left(\Theta_{i}^{a}(\varphi)\right)=1$.

Proof. By the construction of the canonical model and Lemma 2.8, $\neg B_{i}(a, \varphi) \in \Gamma \Rightarrow \Gamma \in$ $\Theta_{i}^{a}(\varphi)$, so $X\left(\neg B_{i}(a, \varphi)\right) \subseteq \Theta_{i}^{a}(\varphi)$. Since $\neg B_{i}(a, \varphi) \rightarrow B_{i}\left(1, \neg B_{i}(a, \varphi)\right)$, so $\neg B_{i}(a, \varphi) \in \Gamma \Rightarrow$ $B_{i}\left(1, \neg B_{i}(a, \varphi)\right) \in \Gamma$, thereby $\mu_{i, \Gamma}\left(X\left(\neg B_{i}(a, \varphi)\right)\right)=\sup \left(\left\{b \mid B_{i}\left(b, \neg B_{i}(a, \varphi)\right) \in \Gamma\right\}\right)=1$. For $X\left(\neg B_{i}(a, \varphi)\right) \subseteq \Theta_{i}^{a}(\varphi)$, we have $\mu_{i, \Gamma}\left(\Theta_{i}^{a}(\varphi)\right)=1$ as desired.

Lemma 2.13 For any $\Gamma, P_{i}(\Gamma)$ is a $P B L_{\omega}$-probability space.

Proof. By Lemma 2.4-2.12, it is obvious.

Lemma 2.14 The model $P M$ is a $P B L_{\omega}$-probabilistic model.

Proof. It follows from Lemma 2.4 and Lemma 2.13.

The above lemmas state that the probability space $P_{i}(\Gamma)=\left(S, X_{i, \Gamma}, \mu_{i, \Gamma}\right)$ of the model satisfies all conditions in Definition 2.2, then as a consequence, the model $P M$ is a $P B L_{\omega}$-probabilistic model. In order to get the completeness, we further prove the following lemma, which states that $P M$ is "canonical".

Lemma 2.15 In the model $P M$, for any $\Gamma$ and any $\varphi,(P M, \Gamma) \models \varphi \Leftrightarrow \varphi \in \Gamma$.

Proof. We argue by the cases on the structure of $\varphi$, here we only give the proof in the case of $\varphi \equiv B_{i}(a, \psi)$.

It suffices to prove that: $(P M, \Gamma) \models B_{i}(a, \psi) \Leftrightarrow B_{i}(a, \psi) \in \Gamma$.

If $B_{i}(a, \psi) \in \Gamma$, by the definition of $P M, \mu_{i, \Gamma}(X(\psi))=b \geq a$, therefore $(P M, \Gamma) \models B_{i}(a, \psi)$.

If $B_{i}(a, \psi) \notin \Gamma$, by Lemma 2.8, there exists $b=\sup \left(\left\{c \mid B_{i}(c, \psi) \in \Gamma\right\}\right)$ such that $B_{i}(b, \psi) \in \Gamma$ and $a>b$. By the definition of $P M, \mu_{i, \Gamma}(X(\psi))=b$, therefore $(P M, \Gamma) \not \models B_{i}(a, \psi)$.

Now it is ready to get the completeness of $P B L_{\omega}$ :

Proposition 2.2 (Completeness of $P B L_{\omega}$ ) If $\Gamma \models_{P B L_{\omega}} \varphi$, then $\Gamma \vdash_{P B L_{\omega}} \varphi$. 
Proof. Suppose not, then there is a $P B L_{\omega}$ - consistent formulas set $\Phi=\Gamma \cup\{\neg \varphi\}$, and there is no model $P M$ such that $\Phi$ is satisfied in $P M$. For there is a $P B L_{\omega}$ - maximal consistent formula set $\Sigma$ such that $\Phi \subseteq \Sigma$, by Lemma $2.15, \Phi$ is satisfied in possible world $\Sigma$ of $P M$. It is a contradiction.

Our proof of the above completeness is different from the proof in [3]. The main idea of our proof is to give a canonical model, which can be regarded as a generalization of canonical model method in Kripke semantics. In [3], Fagin and Halpern adopt another technique to get the completeness. Let $\varphi$ be consistent with $A X_{M E A S}$, they show firstly that an $i$-probability formula $\psi \in \operatorname{Sub}^{+}(\varphi)$ is provably equivalent to a formula of the form $\Sigma_{s \in S} c_{S} \mu_{i}\left(\varphi_{s}\right) \geq b$, for some appropriate coefficients $c_{S}$, where $S$ consists of all maximal consistent subsets of $\mathrm{Sub}^{+}(\varphi)$. Then for a fixed agent $i$ and a fixed state $s$, they describe a set of linear equalities and inequalities corresponding to $i$ and $s$, over variables of the form $x_{i s s^{\prime}}$, for $s^{\prime} \in S$. We can think of $x_{i s s^{\prime}}$ as representing $\mu_{i, s}\left(s^{\prime}\right)$, i.e., the probability of state $s^{\prime}$ under agent $i^{\prime}$ s probability distribution at state $s$. Assume that $\psi$ is equivalent to $\Sigma_{s \in S} c_{s} \mu_{i}\left(\varphi_{s}\right) \geq b$. Observe that exactly one of $\psi$ and $\neg \psi$ is in $s$. If $\psi \in s$, then the corresponding inequality is $\Sigma_{s^{\prime} \in S} \mathcal{C}_{\mathcal{S}^{\prime}} x_{i s s^{\prime}} \geq b$. If $\neg \psi \in s$, then the corresponding inequality is $\Sigma_{S^{\prime} \in S} \mathcal{S}_{S^{\prime}} x_{i s s^{\prime}}<b$. Finally, we have the equality $\Sigma_{s^{\prime} \in S} x_{i s s^{\prime}}=1$. As shown in Theorem 2.2 in Fagin et al. [4], since $\varphi_{S}$ is consistent, this set of linear equalities and inequalities has a solution $x_{i s s^{\prime}}^{*}, s^{\prime} \in S$. From their idea, it is clear that their proof depends tightly on the axioms of linear equalities and inequalities, whereas there are no such axioms in our inference system. On the other hand, their proof cannot deal with the case of infinite set of formulas, because in this case, we will get an infinite set of linear equalities and inequalities, which contains infinite variables. But their axioms seem insufficient to describe the existence of solutions of an infinite set of linear equalities and inequalities.

Proposition 2.1 and Proposition 2.2 show that the axioms and inference rules of $P B L_{\omega}$ give us a sound and complete axiomatization for probabilistic belief. Moreover, we can prove the finite model property and decidability of the provability problem for some variants of $P B L_{\omega}$ in the following sections.

It is not difficult to see that Axioms 1-6 and Rules 1-4 are not complete for our model. Because otherwise, the compactness property of $P B L_{\omega}$ holds, but we can give the following example to show that the compactness property fails in $P B L_{\omega}$ : any finite sub set of $\left\{B_{i}(1 / 2, \varphi), B_{i}(2 / 3, \varphi)\right.$, $\left.\ldots, B_{i}(n / n+1, \varphi), \ldots\right\} \cup\left\{\neg B_{i}(1, \varphi)\right\}$ has model, whereas the whole set does not. But we do not know whether Axioms 1-6 and Rules 1-5 are complete for our model, i.e., whether Rule 6 is redundant in the inference system. Although we believe that Rule 6 is not redundant, we have no proof up to now.

\section{3. $\mathrm{PBL}_{f}$ and its inner probabilistic semantics}

As is often the case in modal logics, the ideas in our completeness proof can be extended to get a finite model property. Therefore the question arises whether finite model property holds for $P B L_{\omega}$, i.e., for every consistent formula $\varphi$, whether there is a finite sates model satisfies $\varphi$. Unfortunately, we cannot give a positive or negative answer here. Therefore, we seek for some weak variant of $P B L_{\omega}$ whose finite model property can be proved. We call the variant $P B L_{f}$, its reasoning system is the result of deleting Axiom 6 and Rule 6 from $P B L_{\omega}$. In the semantics of $P B L_{f}$, we assign an inner probability space to every possible world in the model, here "inner" means the measure does not obey the additivity condition, but obeys some weak additivity conditions satisfied by inner probability measure. 
The well formed formulas set $L^{P B L_{f}}$ of $P B L_{f}$ is the same as $L^{P B L_{\omega}}$.

\subsection{Semantics of $P B L_{f}$}

Definition 3.1 An inner probabilistic model $P M$ of $P B L_{f}$ is a tuple $\left(S, \pi, P_{1}, \ldots, P_{n}\right)$, where

(1) $S$ is a nonempty finite set whose elements are called possible worlds or states;

(2) $\pi$ is a map: $S \times$ Prop $\rightarrow$ true, false $\}$, where Prop is an atomic formulas set;

(3) $P_{i}$ is a map, it maps every possible world $s$ to a $P B L_{f}$-probability space $P_{i}(s)=\left(S, X, \mu_{i, s}\right)$. Here $X=\wp(S)$.

$\mu_{i, s}$ is a $P B L_{f}$-inner probability measure assigned to the set $X$, which means $\mu_{i, s}$ satisfies the following conditions:

(a) $0 \leq \mu_{i, \mathrm{~s}}(A) \leq 1$ for all $A \in X$.

(b) $\mu_{i, s}(\varnothing)=0$ and $\mu_{i, s}(S)=1$.

(c) If $A_{1}, A_{2} \in X$ and $A_{1} \subseteq A_{2}$, then $\mu_{i, s}\left(A_{1}\right) \leq \mu_{i, s}\left(A_{2}\right)$;

(d) If $A_{1}, A_{2} \in X$ and $A_{1} \cap A_{2}=\varnothing$, then $\mu_{i, s}\left(A_{1} \cup A_{2}\right) \geq \mu_{i, s}\left(A_{1}\right)+\mu_{i, s}\left(A_{2}\right)$;

(e) If $A_{1}, A_{2} \in X$, then $\mu_{i, s}\left(A_{1} \cap A_{2}\right) \geq \mu_{i, s}\left(A_{1}\right)+\mu_{i, s}\left(A_{2}\right)-1$;

(f) Let $\Lambda_{i, s}=\left\{s^{\prime} \mid P_{i}(s)=P_{i}\left(s^{\prime}\right)\right\}$, then $\mu_{i, s}\left(\Lambda_{i, s}\right)=1$.

Remark: Since $X=\wp(S)$, therefore $X$ is a constant set, and we omit the subscript of $X_{i, s}$ as was used in Definition 2.2.

It is easy to see that the conditions (d) and (e) in Definition 3.1 are weaker than the finite additivity condition in Definition 2.2. One can check that if $\mu$ is a probability measure, then inner measure $\mu^{*}$ induced by $\mu$ obeys the conditions (d) and (e) in Definition 3.1, i.e., the reason we call $\mu_{i, s}$ inner probability measure.

The notation $\Lambda_{i, s}$ in the condition (f) represents the set of states whose probability space is same as the probability space of state $s$. Therefore the condition (f) means that for any state $s$, the probability space of almost all states is same as the probability space of $s$.

Definition 3.2 Inner probabilistic semantics of $P B L_{f}$

$(P M, s) \models p$ iff $\pi(s, p)=$ true, where $p$ is an atomic formula;

$(P M, s) \models \neg \varphi$ iff $(P M, s) \not \models \varphi$;

$(P M, s) \models \varphi_{1} \wedge \varphi_{2}$ iff $(P M, s) \models \varphi_{1}$ and $(P M, s) \models \varphi_{2}$;

$(P M, s) \models B_{i}(a, \varphi)$ iff $\mu_{i, s}\left(e v_{P M}(\varphi)\right) \geq a$, where $e v_{P M}(\varphi)=\left\{s^{\prime} \mid\left(P M, s^{\prime}\right) \models \varphi\right\}$.

\subsection{Inference system of $P B L_{f}$}

The inference system of $P B L_{f}$ is the same as $P B L_{\mathscr{W}}$ except without Axiom 6 and Rule 6. Axiom 6 corresponds to the finite additivity property of probability. Since the inner probabilistic measure in the model of $P B L_{f}$ does not obey the finite additivity property, therefore Axiom 6 fails with respect to the semantics of $P B L_{f}$. 


\subsection{Soundness of $P B L_{f}$}

The proof of soundness of $P B L_{f}$ is similar to the proof in Proposition 2.1, but because there are some differences between $P B L_{\omega}$-probabilistic model and $P B L_{f}$-probabilistic model, there are a few differences. For example, in the following proof, we can use the property $\mu_{i, s}\left(A_{1} \cap\right.$ $\left.A_{2}\right) \geq \mu_{i, s}\left(A_{1}\right)+\mu_{i, s}\left(A_{2}\right)-1$ directly, rather than as a corollary of finite additivity property; we apply the property $\mu_{i, s}\left(\Lambda_{i, s}\right)=1$ (where $\Lambda_{i, s}=\left\{s^{\prime} \mid P_{i}(s)=P_{i}\left(s^{\prime}\right)\right\}$ ) in the proof, which also differs from the last property of $P B L_{\omega}$-probabilistic model (If $A \in X_{i, s}$ and $\mu_{i, s}(A) \geq a$, then $\mu_{i, s}\left(\left\{s^{\prime} \mid \mu_{i, s^{\prime}}(A) \geq a\right\}\right)=1$; if $A \in X_{i, s}$ and $\mu_{i, s}(A)<b$, then $\mu_{i, s}\left(\left\{s^{\prime} \mid \mu_{i, s^{\prime}}(A)<b\right\}\right)=1$.).

Proposition 3.1 (Soundness of $P B L_{f}$ ) If $\Gamma \vdash_{P B L_{f}} \varphi$, then $\Gamma \models_{P B L_{f}} \varphi$.

Proof. We only discuss Axiom 2, Axiom 3 and Axiom 4 of $P B L_{f}$, other cases can be proved similarly as in Proposition 2.1.

Axiom 2: Suppose $(P M, s) \models B_{i}(a, \varphi) \wedge B_{i}(b, \psi)$, so $\mu_{i, s}\left(e v_{P M}(\varphi)\right) \geq a$ and $\mu_{i, s}\left(e v_{P M}(\psi)\right) \geq b$. For $\mu_{i, s}$ is $P B L_{f}$-probability measure, we get $\mu_{i, s}\left(e v_{P M}(\varphi \wedge \psi)\right)=\mu_{i, s}\left(e v_{P M}(\varphi) \cap e v_{P M}(\psi)\right) \geq$ $\mu_{i, s}\left(e v_{P M}(\varphi)\right)+\mu_{i, s}\left(e v_{P M}(\psi)\right)-1 \geq a+b-1$, which implies $(P M, s) \models B_{i}(\max (a+b-$ $1,0), \varphi \wedge \psi)$.

Axiom 3: Suppose $(P M, s) \models B_{i}(a, \varphi)$, therefore $\mu_{i, s}\left(e v_{P M}(\varphi)\right) \geq a$. Let $\Lambda_{i, s}=\left\{s^{\prime} \mid P_{i}(s)=\right.$ $\left.P_{i}\left(s^{\prime}\right)\right\}$, then $\Lambda_{i, s} \in X$ and $\mu_{i, s}\left(\Lambda_{i, s}\right)=1$. Let $\Xi=\left\{s^{\prime} \mid \mu_{i, s^{\prime}}\left(e v_{P M}(\varphi)\right) \geq a\right\}$. Since $s^{\prime} \in \Lambda_{i, s}$ implies $s^{\prime} \in \Xi$, it is clear $\Lambda_{i, s} \subseteq \Xi$, since $\mu_{i, s}\left(\Lambda_{i, s}\right)=1$, so $\mu_{i, s}(\Xi)=1$. If $s^{\prime} \in \Xi$, then $s^{\prime} \in e v_{P M}\left(B_{i}(a, \varphi)\right)$, therefore $\mu_{i, s}\left(e v_{P M}\left(B_{i}(a, \varphi)\right)\right)=1$, we get $(P M, s) \models B_{i}\left(1, B_{i}(a, \varphi)\right)$ as desired.

Axiom 4: Suppose $(P M, s) \models \neg B_{i}(a, \varphi)$, so $\mu_{i, s}\left(\operatorname{ev}_{P M}(\varphi)\right)<a$. Let $\Lambda_{i, s}=\left\{s^{\prime} \mid P_{i}(s)=P_{i}\left(s^{\prime}\right)\right\}$, then $\Lambda_{i, s} \in X$ and $\mu_{i, s}\left(\Lambda_{i, s}\right)=1$. Let $\Xi=\left\{s^{\prime} \mid \mu_{i, s^{\prime}}\left(e v_{P M}(\varphi)\right)<a\right\}$, for $s^{\prime} \in \Lambda_{i, s}$ implies $s^{\prime} \in \Xi$, it is clear $\Lambda_{i, s} \subseteq \Xi$, since $\mu_{i, s}\left(\Lambda_{i, s}\right)=1$, so $\mu_{i, s}(\Xi)=1$. If $s^{\prime} \in \Xi$, then $s^{\prime} \in e v_{P M}\left(B_{i}(a, \varphi)\right)$, therefore $\mu_{i, s}\left(\operatorname{ev} P M\left(\neg B_{i}(a, \varphi)\right)\right)=1$, we get $(P M, s) \models B_{i}\left(1, \neg B_{i}(a, \varphi)\right)$ as desired.

\subsection{Finite model property of $P B L_{f}$}

We now turn our attention to the finite model property of $P B L_{f}$. It needs to show that if a formula is $P B L_{f}$-consistent, then it is satisfiable in a finite structure. The idea is that rather than considering maximal consistent formulas set when trying to construct a structure satisfying a formula $\varphi$, we restrict our attention to sets of subformulas of $\varphi$.

Definition 3.3 Suppose $\zeta$ is a consistent formula with respect to $P B L_{f}, S u b^{*}(\zeta)$ is a set of formulas defined as follows: let $\zeta \in L^{P B L_{f}}, S u b(\zeta)$ is the set of subformulas of $\zeta$, then $\operatorname{Sub}^{*}(\zeta)=\operatorname{Sub}(\zeta) \cup\{\neg \psi \mid \psi \in \operatorname{Sub}(\zeta)\}$. It is clear that $\operatorname{Sub} b^{*}(\zeta)$ is finite.

Definition 3.4 The inner probabilistic model $P M_{\zeta}$ with respect to formula $\zeta$ is $\left(S_{\zeta}, P_{1, \zeta}, \ldots, P_{n, \zeta}, \pi_{\zeta}\right)$.

(1) Here $S_{\zeta}=\left\{\Gamma \mid \Gamma\right.$ is a maximal consistent formulas set with respect to $P B L_{f}$ and $\Gamma \subseteq$ $\left.S u b^{*}(\zeta)\right\}$.

(2) For any $\Gamma \in S_{\zeta}, P_{i, \zeta}(\Gamma)=\left(S_{\zeta}, X_{\zeta}, \mu_{\zeta, i, \Gamma}\right)$, where $X_{\zeta}=\left\{X(\varphi) \mid X(\varphi)=\left\{\Gamma^{\prime} \mid \varphi\right.\right.$ is a Boolean combination of formulas in $S u b^{*}(\zeta)$ and $\left.\left.\Gamma \vdash_{P B L_{f}} \varphi\right\}\right\} ; \mu_{\zeta, i, \Gamma}$ is an inner probability assignment: $X_{\zeta} \rightarrow[0,1]$, and $\mu_{\zeta, i, \Gamma}(X(\varphi))=\sup \left(\left\{a \mid B_{i}(a, \varphi)\right.\right.$ is provable from $\Gamma$ in $\left.\left.P B L_{f}\right\}\right)$.

(3) $\pi_{\zeta}$ is a truth assignment as follows: For any atomic formula $p, \pi_{\zeta}(p, \Gamma)=$ true $\Leftrightarrow p \in \Gamma$. 
Similar to the proof of completeness of $P B L_{\omega}$, we mainly need to show that the above canonical model $P M_{\zeta}$ is a $P B L_{f}$-inner probabilistic model. The following lemmas from Lemma 3.1 to Lemma 3.13 contribute to this purpose. Furthermore, Lemma 3.14 states that $P M_{\zeta}$ is "canonical", i.e., for any consistent formula $\varphi \in S u b^{*}(\zeta)$, there is a state $s$, such that $\left(P M_{\zeta}, s\right) \models \varphi$. Since we can prove that $P M_{\zeta}$ is a finite model, these lemmas imply the finite model property of $P B L_{f}$.

Lemma $3.1 S_{\zeta}$ is a nonempty finite set.

Proof. Since the rules and axioms of $P B L_{f}$ are consistent, $S_{\zeta}$ is nonempty. For $S u b^{*}(\zeta)$ is a finite set, by the definition of $S_{\zeta}$, the cardinality of $S_{\zeta}$ is no more than the cardinality of $\wp\left(S u b^{*}(\zeta)\right)$.

Lemma $3.2 X_{\zeta}$ is the power set of $S_{\zeta}$.

Proof. Firstly, since $S u b^{*}(\zeta)$ is finite, so if $\Gamma \in S_{\zeta}$ then $\Gamma$ is finite. We can let $\varphi_{\Gamma}$ be the conjunction of the formulas in $\Gamma$. Secondly, if $A \subseteq S_{\zeta}$, then $A=X\left(\vee_{\Gamma \in A} \varphi_{\Gamma}\right)$. By the above argument, we have that $X_{\zeta}$ is the power set of $S_{\zeta}$.

Lemma 3.3 If $\varphi$ is consistent (here $\varphi$ is a Boolean combination of formulas in $S u b^{*}(\zeta)$ ), then there exists $\Gamma$ such that $\varphi$ can be proved from $\Gamma$, here $\Gamma$ is a maximal consistent set with respect to $P B L_{f}$ and $\Gamma \subseteq S u b^{*}(\zeta)$.

Proof. For $\varphi$ is a Boolean combination of formulas in $S u b^{*}(\zeta)$, therefore by regarding the formulas in $S u b^{*}(\zeta)$ as atomic formulas, $\varphi$ can be represented as disjunctive normal form. Since $\varphi$ is consistent, so there is a consistent disjunctive term in disjunctive normal form expression of $\varphi$, let such term be $\psi_{1} \wedge \ldots \wedge \psi_{n}$, then $\varphi$ can be derived from the maximal consistent set $\Gamma$ which contains $\left\{\psi_{1}, \ldots, \psi_{n}\right\}$.

Lemma 3.4 For any $\Gamma \in S_{\zeta}, P_{i, \zeta}(\Gamma)$ is well defined.

Proof. It suffices to prove the following claim: if $X(\varphi)=X(\psi)$, then $\mu_{\zeta, i, \Gamma}(X(\varphi))=$ $\mu_{\zeta, i, \Gamma}(X(\psi))$. If $X(\varphi)=X(\psi)$, it is clear that $\vdash \varphi \leftrightarrow \psi$. For suppose not, then $\varphi \wedge \neg \psi$ is consistent, by Lemma 3.3, there is $\Gamma^{\prime}$ such that $\varphi \wedge \neg \psi$ can be proved from $\Gamma^{\prime}$, therefore $\Gamma^{\prime} \in X(\varphi)$ and $\Gamma^{\prime} \notin X(\psi)$, it is a contradiction. Thus $\vdash \varphi \leftrightarrow \psi$. By rule: $\vdash \varphi \rightarrow \psi \Rightarrow$ $\vdash B_{i}(a, \varphi) \rightarrow B_{i}(a, \psi)$, we get $\vdash B_{i}(a, \varphi) \leftrightarrow B_{i}(a, \psi)$, which means $\mu_{\zeta, i, \Gamma}(X(\varphi))=\mu_{\zeta, i, \Gamma}(X(\psi))$.

Lemma 3.5 Let $\operatorname{Pro}_{\zeta, i, \Gamma}(\varphi)=\left\{a \mid B_{i}(a, \varphi)\right.$ can be proved from $\Gamma$ in $\left.P B L_{f}\right\}$, then $\sup \left(\operatorname{Pro}_{\zeta, i, \Gamma}(\varphi)\right) \in \operatorname{Pro}_{\zeta, i, \Gamma}(\varphi)$.

Proof. Suppose $\operatorname{Pro}_{\zeta, i, \Gamma}(\varphi)=\left\{a \mid B_{i}(a, \varphi)\right.$ can be proved from $\Gamma$ in $\left.P B L_{f}\right\}$, therefore $\Gamma \vdash$ $B_{i}\left(a_{n}, \varphi\right)$ for all $a_{n} \in \operatorname{Pro}_{\zeta, i, \Gamma}(\varphi)$, by Rule 5 of $P B L_{f}, \Gamma \vdash B_{i}(a, \varphi)$, where $a=\sup _{n \in M}\left(\left\{a_{n}\right\}\right)=$ $\sup \left(\operatorname{Pro}_{\zeta, i, \Gamma}(\varphi)\right)$, so we get $\sup \left(\operatorname{Pro}_{\zeta, i, \Gamma}(\varphi)\right) \in \operatorname{Pro}_{\zeta, i, \Gamma}(\varphi)$ as desired.

Lemma 3.6 If $A \in X_{\zeta}$, then $0 \leq \mu_{\zeta, i, \Gamma}(A) \leq 1$. Furthermore, $\mu_{\zeta, i, \Gamma}(\varnothing)=0, \mu_{\zeta, i, \Gamma}\left(S_{\zeta}\right)=1$.

Proof. By the definition, if $B_{i}(a, \varphi)$ is a well formed formula, then $0 \leq a \leq 1$; furthermore, check the axioms and rules of $P B L_{f}$, any formula derived from well formed formulas is also a well formed formula, so $0 \leq \mu_{\zeta, i, \Gamma}(X(\varphi)) \leq 1$. Therefore, if $A \in X_{\zeta}$, then $0 \leq \mu_{\zeta, i, \Gamma}(A) \leq 1$.

By rule: $\vdash \varphi \Rightarrow \vdash B_{i}(1, \varphi)$, therefore we have $\mu_{\zeta, i, \Gamma}\left(S_{\zeta}\right)=1$ as desired. By axiom: $B_{i}(0, \varphi)$, we get $B_{i}(0$, false $)$, so $\mu_{\zeta, i, \Gamma}(\varnothing) \geq 0$. By rule: $\vdash \neg(\varphi \wedge \psi) \Rightarrow \vdash B_{i}(a, \varphi) \wedge B_{i}(b, \psi) \rightarrow B_{i}(a+$ $b, \varphi \vee \psi)$, where $a+b \leq 1$, we have $\vdash B_{i}($ a, false $) \wedge B_{i}(b$, true $) \rightarrow B_{i}(a+b$, false $\vee$ true $)$, 
hence $\mu_{\zeta, i, \Gamma}\left(S_{\zeta}\right) \geq \mu_{\zeta, i, \Gamma}\left(S_{\zeta}\right)+\mu_{\zeta, i, \Gamma}(\varnothing)$. Since $\mu_{\zeta, i, \Gamma}\left(S_{\zeta}\right)=1$, so $1 \geq 1+\mu_{\zeta, i, \Gamma}(\varnothing)$, therefore $\mu_{\zeta, i, \Gamma}(\varnothing)=0$ as desired.

Lemma 3.7 If $A_{1}, A_{2} \in X_{\zeta}$ and $A_{1} \subseteq A_{2}$, then $\mu_{\zeta, i, \Gamma}\left(A_{1}\right) \leq \mu_{\zeta, i, \Gamma}\left(A_{2}\right)$.

Proof. Since $A_{1}, A_{2} \in X_{\zeta}$, assume $A_{1}=X(\varphi), A_{2}=X(\psi)$. If $X(\varphi) \subseteq X(\psi)$, by rule: $\vdash \varphi \rightarrow \psi$ $\Rightarrow \vdash B_{i}(a, \varphi) \rightarrow B_{i}(a, \psi)$, we have $\mu_{\zeta, i, \Gamma}(X(\varphi)) \leq \mu_{\zeta, i, \Gamma}(X(\psi))$. Therefore if $A_{1}, A_{2} \in X_{\zeta}$ and $A_{1} \subseteq A_{2}$, then $\mu_{\zeta, i, \Gamma}\left(A_{1}\right) \leq \mu_{\zeta, i, \Gamma}\left(A_{2}\right)$.

Lemma 3.8 If $A_{1}, A_{2} \in X_{\zeta}$ and $A_{1} \cap A_{2}=\varnothing$, then $\mu_{\zeta, i, \Gamma}\left(A_{1} \cup A_{2}\right) \geq \mu_{\zeta, i, \Gamma}\left(A_{1}\right)+\mu_{\zeta, i, \Gamma}\left(A_{2}\right)$.

Proof. Since $A_{1}, A_{2} \in X_{\zeta}$, assume $A_{1}=X(\varphi), A_{2}=X(\psi)$, by rule: $\vdash \neg(\varphi \wedge \psi) \Rightarrow \vdash B_{i}(a, \varphi) \wedge$ $B_{i}(b, \psi) \rightarrow B_{i}(a+b, \varphi \vee \psi)$, where $a_{1}+a_{2} \leq 1$, we have $\mu_{\zeta, i, \Gamma}(X(\varphi) \cup X(\psi)) \geq \mu_{\zeta, i, \Gamma}(X(\varphi))+$ $\mu_{\zeta, i, \Gamma}(X(\psi))$. Therefore if $A_{1}, A_{2} \in X_{\zeta}$ and $A_{1} \cap A_{2}=\varnothing$, then $\mu_{\zeta, i, \Gamma}\left(A_{1} \cup A_{2}\right) \geq \mu_{\zeta, i, \Gamma}\left(A_{1}\right)+$ $\mu_{\zeta, i, \Gamma}\left(A_{2}\right)$.

Lemma 3.9 For any $C, D \in X_{\zeta}, \mu_{\zeta, i, \Gamma}(C \cap D) \geq \mu_{\zeta, i, \Gamma}(C)+\mu_{\zeta, i, \Gamma}(D)-1$.

Proof. Since $C, D \in X_{\zeta}$, assume $C=X(\varphi), D=X(\psi)$, by axiom: $B_{i}(a, \varphi) \wedge B_{i}(b, \psi) \rightarrow$ $B_{i}(\max (a+b-1,0), \varphi \wedge \psi)$, we get $\mu_{\zeta, i, \Gamma}(X(\varphi) \cap X(\psi)) \geq \mu_{\zeta, i, \Gamma}(X(\varphi))+\mu_{\zeta, i, \Gamma}(X(\psi))-1$.

Lemma 3.10 Let $B_{i}^{-}(\Gamma)=\left\{\Gamma^{\prime} \mid\left\{\varphi: B_{i}(1, \varphi) \in \Gamma\right\} \subseteq \Gamma^{\prime}\right\}$, then $\mu_{\zeta, i, \Gamma}\left(B_{i}^{-}(\Gamma)\right)=1$.

Proof. For $\Gamma$ is a finite formulas set, therefore $B_{i}^{-}(\Gamma)=X\left(\wedge_{B_{i}\left(1, \varphi_{n}\right) \in \Gamma} \varphi_{n}\right)$, by axiom: $B_{i}(a, \varphi) \wedge B_{i}(b, \psi) \rightarrow B_{i}(\max (a+b-1,0), \varphi \wedge \psi)$, we have that $\wedge B_{i}\left(1, \varphi_{n}\right) \rightarrow B_{i}\left(1, \wedge \varphi_{n}\right)$, so $B_{i}\left(1, \wedge_{B_{i}}\left(1, \varphi_{n}\right) \in \Gamma \varphi_{n}\right)$ can be proved from $\Gamma$ in $P B L_{f}$, so $\mu_{\zeta, i, \Gamma}\left(B_{i}^{-}(\Gamma)\right)=1$.

Lemma 3.11 Let $\Lambda_{i, \Gamma}=\left\{\Gamma^{\prime} \mid P_{i, \zeta}(\Gamma)=P_{i, \zeta}\left(\Gamma^{\prime}\right)\right\}$, then $\mu_{\zeta, i, \Gamma}\left(\Lambda_{i, \Gamma}\right)=1$.

Proof. Suppose $\Gamma^{\prime} \in B_{i}^{-}(\Gamma)$. If $B_{i}(a, \varphi) \in \Gamma$, by rule: $B_{i}(a, \varphi) \rightarrow B_{i}\left(1, B_{i}(a, \varphi)\right)$, we get $B_{i}\left(1, B_{i}(a, \varphi)\right) \in \Gamma$, for $\Gamma^{\prime} \in B_{i}^{-}(\Gamma)$, hence $B_{i}(a, \varphi) \in \Gamma^{\prime}$. If $\neg B_{i}(a, \varphi) \in \Gamma$, by rule: $\neg B_{i}(a, \varphi) \rightarrow$ $B_{i}\left(1, \neg B_{i}(a, \varphi)\right)$, we get $B_{i}\left(1, \neg B_{i}(a, \varphi)\right) \in \Gamma$, for $\Gamma^{\prime} \in B_{i}^{-}(\Gamma)$, hence $\neg B_{i}(a, \varphi) \in \Gamma^{\prime}$. Therefore $B_{i}(a, \varphi) \in \Gamma$ iff $B_{i}(a, \varphi) \in \Gamma^{\prime}$, which means for any $A \in X_{\zeta}, \mu_{\zeta, i, \Gamma}(A)=\mu_{\zeta, i, \Gamma^{\prime}}(A)$, so $\Gamma^{\prime} \in \Lambda_{i, \Gamma}$, and furthermore $B_{i}^{-}(\Gamma) \subseteq \Lambda_{i, \Gamma}$. By Lemma 3.10, $\mu_{\zeta, i, \Gamma}\left(B_{i}^{-}(\Gamma)\right)=1$, we get $\mu_{\zeta, i, \Gamma}\left(\Lambda_{i, \Gamma}\right)=1$ as desired.

Lemma 3.12 For any $\Gamma \in S_{\zeta}, P_{i, \zeta}(\Gamma)$ is a $P B L_{f}$-inner probability space.

Proof. By Lemma 3.6 to Lemma 3.11, we can get the claim immediately.

Lemma 3.13 The inner probabilistic model $P M_{\zeta}$ is a finite model.

Proof. By the definition of $S_{\zeta}$, the cardinality of $S_{\zeta}$ is no more than the cardinality of $\wp\left(S u b^{*}(\zeta)\right)$, which means $\left|S_{\zeta}\right| \leq 2^{\left|S u b^{*}(\zeta)\right|}$.

Similar to the proof of completeness of $P B L_{\omega}$, the above lemmas show that $P M_{\zeta}$ is a finite $P B L_{f}$-model and the following lemma states that $P M_{\zeta}$ is canonical.

Lemma 3.14 For the finite canonical model $P M_{\zeta}$, for any $\Gamma \in S_{\zeta}$ and any $\varphi \in S u b^{*}(\zeta)$, $\left(P M_{\zeta}, \Gamma\right) \models \varphi \Leftrightarrow \varphi \in \Gamma$.

Proof. We argue by cases on the structure of $\varphi$, here we only give the proof in the case of $\varphi \equiv B_{i}(a, \psi)$ :

It suffices to prove: $\left(P M_{\zeta}, \Gamma\right) \models B_{i}(a, \psi) \Leftrightarrow B_{i}(a, \psi) \in \Gamma$. 
If $B_{i}(a, \psi) \in \Gamma$, by the definition of $P M_{\zeta}, \mu_{\zeta, i, \Gamma}(X(\psi))=b \geq a$, therefore $\left(P M_{\zeta}, \Gamma\right) \models B_{i}(a, \psi)$.

If $B_{i}(a, \psi) \notin \Gamma$, by Lemma 3.5, there exists $b=\sup \left(\left\{c \mid B_{i}(c, \psi) \in \Gamma\right\}\right)$ such that $B_{i}(b, \psi) \in \Gamma$ and $a>b$. By the definition of $P M_{\zeta}, \mu_{\zeta, i, \Gamma}(X(\psi))=b$, therefore $\left(P M_{\zeta}, \Gamma\right) \not \models B_{i}(a, \psi)$.

From the above lemmas, we know that $P M_{\zeta}$ is a finite $P B L_{f}$-model that is canonical. Now it is no difficult to get the following proposition.

Proposition 3.2 (Finite model property of $P B L_{f}$ ) If $\Gamma$ is a finite set of consistent formulas, then there is a finite $P B L_{f}$-model $P M$ such that $P M \models_{P B L_{f}} \Gamma$.

Proof. By Lemma 3.14, there exists a finite $P B L_{f}$-model $P M_{\wedge \Gamma}$ such that $\Gamma$ is satisfied in $P M_{\wedge \Gamma}$.

Proposition 3.3 (Weak completeness of $P B L_{f}$ ) If $\Gamma$ is a finite set of formulas, $\varphi$ is a formula, and $\Gamma \models{ }_{P B L_{f}} \varphi$, then $\Gamma \vdash_{P B L_{f}} \varphi$.

Proof. Suppose not, then $(\wedge \Gamma) \wedge \neg \varphi$ is consistent with respect to $P B L_{f}$, by Proposition 3.2, there exists an inner probabilistic model $P M_{(\wedge \Gamma) \wedge \neg \varphi}$ such that $(\wedge \Gamma) \wedge \neg \varphi$ is satisfied in $P M_{(\wedge \Gamma) \wedge \neg \varphi}$, but this contradicts our assumption that $\Gamma \models{ }_{P B L_{f}} \varphi$, thus the proposition holds.

As to $P B L_{\omega}$ case, the construction of canonical model like Definition 3.4 fails to get the finite model property. The main problem lies in how to define measure assignment $\mu_{\zeta, i, \Gamma}$, in Definition 3.4, $\mu_{\zeta, i, \Gamma}(X(\varphi))=\sup \left(\left\{a \mid B_{i}(a, \varphi)\right.\right.$ is provable from $\Gamma$ in $\left.\left.P B L_{f}\right\}\right)$, but Rule 6 fails under this definition. Thus there is an unsolved problem about how to construct a finite model with respect to a $P B L_{\omega}$-consistent formula.

Usually, in the case of modal logics, one can get decidability of the provability problem from finite model property. At first, one can simply construct every model with finite (for example, say $2^{\left|S u b^{*}(\varphi)\right|}$ ) states. One then check if $\varphi$ is true at some state of one of these models (note that the number of models that have $2^{\left|S u b^{*}(\varphi)\right|}$ states is finite). By finite model property, if a formula $\varphi$ is consistent, then $\varphi$ is satisfiable with respect to some models. Conversely, if $\varphi$ is satisfiable with respect to some models, then $\varphi$ is consistent.

But it becomes different for $P B L_{f}$. Because there may be infinitely many $P B L_{f}$-inner probability measure assigned to the set $X$ (since real number in $[0,1]$ is infinite), there are infinitely many probabilistic models associated to a given number of states (for example, say $\left.2^{\left|S u b^{*}(\varphi)\right|}\right)$. Therefore the above argument fails. On the contrary, in the next section, we will present another variant- $P B L_{r}$, and prove that the decidability of the provable problem holds for $P B L_{r}$.

\section{4. $P B L_{r}$ and its inner probabilistic semantics}

The inference systems of $P B L_{\omega}$ and $P B L_{f}$ both have the infinite inference rules, but in application, an infinite inference rule is inconvenient. Whether we can get the weak completeness for a variant of $P B L_{\omega}$ or $P B L_{f}$ without Rule 5? In this section, we propose another probabilistic belief logic- $P B L_{r}$. The inference system of $P B L_{r}$ is that of $P B L_{f}$ without Rule 5. Another notable difference between $P B L_{r}$ and $P B L_{f}$ is that the probability $a$ in the scope of $B_{i}(a, \varphi)$ must be a rational number. Similar to the semantics of $P B L_{f}$, we assign an inner probability space to every possible world in the model.

We prove the soundness and finite model property of $P B L_{r}$. At last, as a consequence of the finite model property, we obtain weak completeness and decidability of the provability 
problem of $P B L_{f}$. Roughly speaking, let $\Gamma$ be a finite set of formulas, weak completeness means $\Gamma \models \varphi \Rightarrow \Gamma \vdash \varphi$, and decidability of the provability problem of $P B L_{f}$ means there is an algorithm that, given as input a formula $\varphi$, will decide whether $\varphi$ is provable in $P B L_{f}$.

Definition 4.1 The set of well formed formulas set of $P B L_{r}$, called $L^{P B L_{r}}$, is given by the following grammar:

(1) If $\varphi \in$ Atomic formulas set, then $\varphi \in L^{P B L_{r}}$;

(2) If $\varphi \in L^{P B L_{r}}$, then $\neg \varphi \in L^{P B L_{r}}$;

(3) If $\varphi_{1}, \varphi_{2} \in L^{P B L_{r}}$, then $\varphi_{1} \wedge \varphi_{2} \in L^{P B L_{r}}$;

(4) If $\varphi \in L^{P B L_{r}}$ and $a$ is a rational number in [0,1], then $B_{i}(a, \varphi) \in L^{P B L_{r}}$.

Remark: A significant difference between $P B L_{r}$ and $P B L_{\mathcal{O}}\left(P B L_{f}\right)$ is that in the definition of syntax, the probability in the scope of $B_{i}(a, \varphi)$ in the former is a rational number.

The inner probabilistic model of $P B L_{r}$ is the same as the inner probabilistic model of $P B L_{f}$, except that the value of $P B L_{r}$-inner probability measure is a rational number.

The inference system of $P B L_{r}$ consists of axioms and inference rules of proposition logic and the Axioms 1-5 and Rules 1-4 of $P B L_{\mathscr{O}}$. But it is necessary to note that by the definition of well formed formulas of $P B L_{r}$, all the probabilities in the axioms and inference rules of $P B L_{r}$ should be modified to be rational numbers. For example, Axiom 5 of $P B L_{\omega}$ : " $B_{i}(a, \varphi) \rightarrow B_{i}(b, \varphi)$, where $1 \geq a \geq b \geq 0$ " should be modified as " $B_{i}(a, \varphi) \rightarrow B_{i}(b, \varphi)$, where $1 \geq a \geq b \geq$ 0 and $a, b$ are rational numbers" in $P B L_{r}$. Since the probabilities $a$ and $b$ in the formulas $B_{i}(a, \varphi)$ and $B_{i}(b, \psi)$ are rational numbers, so the probability $\max (a+b-1,0)$ in the scope of $B_{i}(\max (a+b-1,0), \varphi \wedge \psi)$ in Axiom 2 and the probability $a+b$ in the scope of $B_{i}(a+b, \varphi \vee \psi)$ in Rule 4 are also rational numbers.

The proof of the soundness of $P B L_{r}$ is similar to the soundness of $P B L_{f}$, and we do not give the details.

Proposition 4.1 (Soundness of $P B L_{r}$ ) If $\Gamma \vdash_{P B L_{r}} \varphi$ then $\Gamma \models_{P B L_{r}} \varphi$.

\subsection{Finite model property and decidability of $P B L_{r}$}

In order to prove the weak completeness of $P B L_{r}$, we first present a probabilistic belief logic $P B L_{r}(N)$, where $N$ is a given natural number. The finite model property of $P B L_{r}(N)$ is then proved. From this property, we get the weak completeness and the decidability of $P B L_{r}$.

The syntax of $P B L_{r}(N)$ is the same as the syntax of $P B L_{r}$ except that the probabilities in formulas should be rational numbers like $k / N$. For example, every probability in formulas of $P B L_{r}(3)$ should be one of $0 / 3,1 / 3,2 / 3$ or $3 / 3$. Therefore, $B_{i}(1 / 3, \varphi)$ and $B_{i}\left(2 / 3, B_{j}(1 / 3, \varphi)\right)$ are well formed formulas in $P B L_{r}(3)$, but $B_{i}(1 / 2, \varphi)$ is not a well formed formula in $P B L_{r}(3)$.

The inner probabilistic model of $P B L_{r}(N)$ is also the same as $P B L_{r}$ except that the measure assigned to every possible world should be the form of $k / N$ respectively. Therefore, in an inner probabilistic model of $P B L_{r}(3)$, the measure in a possible world may be $1 / 3,2 / 3$ and etc, but can not be $1 / 2$ or $1 / 4$.

The inference system of $P B L_{r}(N)$ is also similar to $P B L_{r}$ but all the probabilities in the axioms and inference rules should be the form of $k / N$ respectively. For example, Axiom 5 of $P B L_{\omega}$ : 
" $B_{i}(a, \varphi) \rightarrow B_{i}(b, \varphi)$, where $1 \geq a \geq b \geq 0$ " should be modified to " $B_{i}(a, \varphi) \rightarrow B_{i}(b, \varphi)$, where $1 \geq a \geq b \geq 0$ and $a, b$ are the from of $k_{1} / N, k_{2} / N^{\prime \prime}$ in Axiom 5 of the inference system of $P B L_{r}(N)$. Since the probabilities $a$ and $b$ in the formulas $B_{i}(a, \varphi)$ and $B_{i}(b, \varphi)$ are in the form of $k_{1} / N, k_{2} / N$, so the probability $\max (a+b-1,0)$ in the scope of $B_{i}(\max (a+b-1,0), \varphi \wedge \psi)$ in Axiom 2 and the probability $a+b$ in the scope of $B_{i}(a+b, \varphi \vee \psi)$ in Rule 4 are also in the form of $k / N$.

It is easy to see that the soundness of $P B L_{r}(N)$ holds. We omit the detail proof here.

Proposition 4.2 (Soundness of $\left.P B L_{r}(N)\right)$ If $\Gamma \vdash_{P B L_{r}(N)} \varphi$ then $\Gamma \models_{P B L_{r}(N)} \varphi$.

In the following, we prove the finite model property of $P B L_{r}(N)$. By this proposition, we can obtain the weak completeness of $P B L_{r}$ immediately.

Definition 4.2 Suppose $\zeta$ is a consistent formula with respect to $P B L_{r}(N)$. Sub $(\zeta)$ is a set of formulas defined as follows: let $\zeta \in L^{P B L_{r}(N)}, \operatorname{Sub}(\zeta)$ is the set of subformulas of $\zeta$, then $\operatorname{Sub}^{*}(\zeta)=\operatorname{Sub}(\zeta) \cup\{\neg \psi \mid \psi \in \operatorname{Sub}(\zeta)\}$. It is clear that $S u b^{*}(\zeta)$ is finite.

Definition 4.3 The inner probabilistic model $P M_{\zeta}$ with respect to formula $\zeta$ is $\left(S_{\zeta}, P_{1, \zeta}, \ldots, P_{n, \zeta}, \pi_{\zeta}\right)$.

(1) Here $S_{\zeta}=\left\{\Gamma \mid \Gamma\right.$ is a maximal consistent formulas set with respect to $P B L_{r}(N)$ and $\Gamma \subseteq$ $\left.\mathrm{Sub}^{*}(\zeta)\right\}$.

(2) For any $\Gamma \in S_{\zeta}, P_{i, \zeta}(\Gamma)=\left(S_{\zeta}, X_{\zeta}, \mu_{\zeta, i, \Gamma}\right)$, where $X_{\zeta}=\left\{X(\varphi) \mid X(\varphi)=\left\{\Gamma^{\prime} \mid \varphi\right.\right.$ is a Boolean combination of formulas in $S_{u b}{ }^{*}(\zeta)$ and $\left.\left.\Gamma^{\prime} \vdash_{P B L_{r}(N)} \varphi\right\}\right\} ; \mu_{\zeta, i, \Gamma}$ is an inner probability assignment: $X_{\zeta} \rightarrow[0,1]$, and $\mu_{\zeta, i, \Gamma}\left(X(\varphi)=\sup \left(\left\{a \mid B_{i}(a, \varphi)\right.\right.\right.$ is provable from $\Gamma$ in $\left.\left.P B L_{r}(N)\right\}\right)$.

(3) $\pi_{\zeta}$ is a truth assignment as follows: for any atomic formula $p, \pi_{\zeta}(p, \Gamma)=$ true $\Leftrightarrow p \in \Gamma$.

The following lemmas show that the above model $P M_{\zeta}$ is an inner probabilistic model of $P B L_{r}(N)$, and it is canonical: for any $\Gamma \in S_{\zeta}$ and any $\varphi \in S u b^{*}(\zeta), \varphi \in \Gamma \Leftrightarrow\left(P M_{\zeta}, \Gamma\right) \models \varphi$. This implies the finite model property of $P B L_{r}(N)$.

Lemma $4.1 S_{\zeta}$ is a nonempty finite set.

Proof. Since the rules and axioms of $P B L_{r}(N)$ are consistent, $S_{\zeta}$ is nonempty. For $S u b^{*}(\zeta)$ is a finite set, by the definition of $S_{\zeta}$, the cardinality of $S_{\zeta}$ is no more than the cardinality of $\wp\left(S u b^{*}(\zeta)\right)$.

Lemma $4.2 X_{\zeta}$ is the power set of $S_{\zeta}$.

Proof. Firstly, since $S u b^{*}(\zeta)$ is finite, so if $\Gamma \in S_{\zeta}$ then $\Gamma$ is finite. We can let $\varphi_{\Gamma}$ be the conjunction of the formulas in $\Gamma$. Secondly, if $A \subseteq S_{\zeta}$, then $A=X\left(\vee_{\Gamma \in A} \varphi_{\Gamma}\right)$. By the above argument, we have that $X_{\zeta}$ is the power set of $S_{\zeta}$.

Lemma 4.3 If $\varphi$ is consistent (here $\varphi$ is a Boolean combination of formulas in $S u b^{*}(\zeta)$ ), then there exists $\Gamma$ such that $\varphi$ can be proved from $\Gamma$, here $\Gamma$ is a maximal consistent set with respect to $P B L_{r}(N)$ and $\Gamma \subseteq S u b^{*}(\zeta)$.

Proof. For $\varphi$ is obtainable from the Boolean connective composition of formulas in $\operatorname{Sub}^{*}(\zeta)$, therefore by regarding the formulas in $S u b^{*}(\zeta)$ as atomic formulas, $\varphi$ can be represented in disjunctive normal form. Since $\varphi$ is consistent, there is a consistent disjunctive term in disjunctive normal form expression of $\varphi$, let such term be $\psi_{1} \wedge \ldots \wedge \psi_{n}$, then $\varphi$ can be derived from the maximal consistent set $\Gamma$ that contains $\left\{\psi_{1}, \ldots, \psi_{n}\right\}$. 
Lemma 4.4 For any $\Gamma \in S_{\zeta}, P_{\zeta}(\Gamma)$ is well defined.

Proof. It suffices to prove the following claim: if $X(\varphi)=X(\psi)$, then $\mu_{\zeta, i, \Gamma}(X(\varphi))=$ $\mu_{\zeta, i, \Gamma}(X(\psi))$. If $X(\varphi)=X(\psi)$, it is clear that $\vdash \varphi \leftrightarrow \psi$. For suppose not, $\varphi \wedge \neg \psi$ is consistent. By Lemma 4.3, there is $\Gamma^{\prime}$ such that $\varphi \wedge \neg \psi$ can be proved from $\Gamma^{\prime}$, therefore $\Gamma^{\prime} \in X(\varphi)$ and $\Gamma^{\prime} \notin X(\psi)$, it is a contradiction. Thus $\vdash \varphi \leftrightarrow \psi$. By rule: $\vdash \varphi \rightarrow \psi \Rightarrow \vdash B_{i}(a, \varphi) \rightarrow B_{i}(a, \psi)$, we get $\vdash B_{i}(a, \varphi) \leftrightarrow B_{i}(a, \psi)$, which means $\mu_{\zeta, i, \Gamma}(X(\varphi))=\mu_{\zeta, i, \Gamma}(X(\psi))$.

Lemma 4.5 Let $\operatorname{Pro}_{\zeta, i, \Gamma}(\varphi)=\left\{a \mid B_{i}(a, \varphi) \in \Gamma\right\}$, then $\sup \left(\operatorname{Pro}_{\zeta, i, \Gamma}(\varphi)\right) \in \operatorname{Pro}_{\zeta, i, \Gamma}(\varphi)$.

Proof. By the construction of model, $\operatorname{Pro}_{\zeta, i, \Gamma}(\varphi)$ is one of the numbers $0 / N, 1 / N, \ldots, N / N$. Since the set $0 / N, 1 / N, \ldots, N / N$ is finite, therefore $\sup \left(\operatorname{Pro}_{\zeta, i, \Gamma}(\varphi)\right) \in \operatorname{Pro}_{\zeta, i, \Gamma}(\varphi)$.

Lemma 4.6 If $A \in X_{\zeta}$, then $0 \leq \mu_{\zeta, i, \Gamma}(A) \leq 1$. Furthermore, $\mu_{\zeta, i, \Gamma}(\varnothing)=0$ and $\mu_{\zeta, i, \Gamma}\left(S_{\zeta}\right)=1$.

Proof. By the construction of model, it is clear that $\mu_{\zeta, i, \Gamma}$ has the following property: if $A \in X_{\zeta}$, then $0 \leq \mu_{\zeta, i, \Gamma}(A) \leq 1$.

By rule: $\vdash \varphi \Rightarrow \vdash B_{i}(1, \varphi)$, it is clear $\mu_{\zeta, i, \Gamma}\left(S_{\zeta}\right)=1$. By axiom: $B_{i}(0, \varphi)$, we get $B_{i}(0$, false $)$, so $\mu_{\zeta, i, \Gamma}(\varnothing) \geq 0$. By Rule 4 of $P B L_{r}$, we get $\mu_{\zeta, i, \Gamma}\left(S_{\zeta}\right) \geq \mu_{\zeta, i, \Gamma}\left(S_{\zeta}\right)+\mu_{\zeta, i, \Gamma}(\varnothing)$, so $1 \geq 1+\mu_{\zeta, i, \Gamma}(\varnothing)$, which implies $\mu_{\zeta, i, \Gamma}(\varnothing)=0$.

Lemma 4.7 If $A_{1}, A_{2} \in X_{\zeta}$ and $A_{1} \subseteq A_{2}$, then $\mu_{\zeta, i, \Gamma}\left(A_{1}\right) \leq \mu_{\zeta, i, \Gamma}\left(A_{2}\right)$.

Proof. Since $A_{1}, A_{2} \in X_{\zeta}$ assume $A_{1}=X(\varphi), A_{2}=X(\psi)$. If $X(\varphi) \subseteq X(\psi)$, by rule: $\vdash \varphi \rightarrow$ $\psi \Rightarrow \vdash B_{i}(a, \varphi) \rightarrow B_{i}(a, \psi)$, we have $\mu_{\zeta, i, \Gamma}\left(A_{1}\right) \leq \mu_{\zeta, i, \Gamma}\left(A_{2}\right)$. Therefore if $A_{1}, A_{2} \in X_{\zeta}$ and $A_{1} \subseteq A_{2}$, then $\mu_{\zeta, i, \Gamma}\left(A_{1}\right) \leq \mu_{\zeta, i, \Gamma}\left(A_{2}\right)$.

Lemma 4.8 If $A_{1}, A_{2} \in X_{\zeta}$ and $A_{1} \cap A_{2}=\varnothing$, then $\mu_{\zeta, i, \Gamma}\left(A_{1} \cup A_{2}\right) \geq \mu_{\zeta, i, \Gamma}\left(A_{1}\right)+\mu_{\zeta, i, \Gamma}\left(A_{2}\right)$.

Proof. Since $A_{1}, A_{2} \in X_{\zeta}$, assume $A_{1}=X(\varphi), A_{2}=X(\psi)$. By rule: $\vdash \neg(\varphi \wedge \psi) \Rightarrow$ $\vdash B_{i}\left(a_{1}, \varphi\right) \wedge B_{i}\left(a_{2}, \psi\right) \rightarrow B_{i}\left(a_{1}+a_{2}, \varphi \vee \psi\right)$, where $a_{1}+a_{2} \leq 1$, we have $\mu_{\zeta, i, \Gamma}(X(\varphi) \cup X(\psi)) \geq$ $\mu_{\zeta, i, \Gamma}(X(\varphi))+\mu_{\zeta, i, \Gamma}(X(\psi))$. Therefore if $A_{1}, A_{2} \in X_{\zeta}$ and $A_{1} \cap A_{2}=\varnothing$, then $\mu_{\zeta, i, \Gamma}\left(A_{1} \cup A_{2}\right) \geq$ $\mu_{\zeta, i, \Gamma}\left(A_{1}\right)+\mu_{\zeta, i, \Gamma}\left(A_{2}\right)$.

Lemma 4.9 For any $\left.C, D \in X_{\zeta}, \mu_{\zeta, i, \Gamma}(C \cap D) \geq \mu_{\zeta, i, \Gamma} C\right)+\mu_{\zeta, i, \Gamma}(D)-1$.

Proof. Since $C, D \in X_{\zeta}$, assume $C=X(\varphi), D=X(\psi)$, by axiom: $B_{i}(a, \varphi) \wedge B_{i}(b, \psi) \rightarrow$ $B_{i}(\max (a+b-1,0), \varphi \wedge \psi)$, we get $\mu_{\zeta, i, \Gamma}(X(\varphi) \cap X(\psi)) \geq \mu_{\zeta, i, \Gamma}(X(\varphi))+\mu_{\zeta, i, \Gamma}(X(\psi))-1$.

Lemma 4.10 Let $B_{i}^{-}(\Gamma)=\left\{\Gamma^{\prime} \mid\left\{\varphi \mid B_{i}(1, \varphi) \in \Gamma\right\} \subseteq \Gamma^{\prime}\right\}$, then $\mu_{\zeta, i, \Gamma}\left(B_{i}^{-}(\Gamma)\right)=1$.

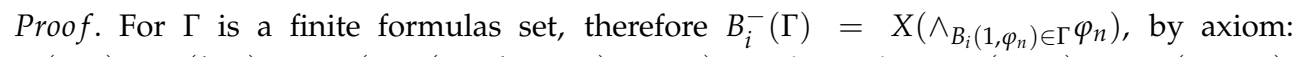
$B_{i}(a, \varphi) \wedge B_{i}(b, \psi) \rightarrow B_{i}(\max (a+b-1,0), \varphi \wedge \psi)$, we have that $\wedge B_{i}\left(1, \varphi_{n}\right) \rightarrow B_{i}\left(1, \wedge \varphi_{n}\right)$, so $B_{i}\left(1, \wedge_{B_{i}\left(1, \varphi_{n}\right) \in \Gamma} \varphi_{n}\right)$ can be proved from $\Gamma$ in $P B L_{r}(N)$, so $\mu_{\zeta, i, \Gamma}\left(B_{i}^{-}(\Gamma)\right)=1$.

Lemma 4.11 Let $\Lambda_{i, \Gamma}=\left\{\Gamma^{\prime} \mid P_{i, \zeta}(\Gamma)=P_{i, \zeta}\left(\Gamma^{\prime}\right)\right\}$, then $\mu_{\zeta, i, \Gamma}\left(\Lambda_{i, \Gamma}\right)=1$.

Proof. Suppose $\Gamma^{\prime} \in B_{i}^{-}(\Gamma)$. If $B_{i}(a, \varphi) \in \Gamma$, by rule: $B_{i}(a, \varphi) \rightarrow B_{i}\left(1, B_{i}(a, \varphi)\right)$, we get $B_{i}\left(1, B_{i}(a, \varphi)\right) \in \Gamma$, for $\Gamma^{\prime} \in B_{i}^{-}(\Gamma)$, hence $B_{i}(a, \varphi) \in \Gamma^{\prime}$. If $\neg B_{i}(a, \varphi) \in \Gamma$, by rule: $\neg B_{i}(a, \varphi) \rightarrow$ $B_{i}\left(1, \neg B_{i}(a, \varphi)\right)$, we get $B_{i}\left(1, \neg B_{i}(a, \varphi)\right) \in \Gamma$, for $\Gamma^{\prime} \in B_{i}^{-}(\Gamma)$, hence $\neg B_{i}(a, \varphi) \in \Gamma^{\prime}$. Therefore $B_{i}(a, \varphi) \in \Gamma$ iff $B_{i}(a, \varphi) \in \Gamma^{\prime}$, which means for any $A \in X_{\zeta}, \mu_{\zeta, i, \Gamma}(A)=\mu_{\zeta, i, \Gamma^{\prime}}(A)$, so $\Gamma^{\prime} \in \Lambda_{i, \Gamma}$, and furthermore $B_{i}^{-}(\Gamma) \subseteq \Lambda_{i, \Gamma}$. For $\mu_{\zeta, i, \Gamma}\left(B_{i}^{-}(\Gamma)\right)=1$, we get $\mu_{\zeta, i, \Gamma}\left(\Lambda_{i, \Gamma}\right)=1$ as desired. 
Lemma 4.12 For any $\Gamma \in S_{\zeta}, P_{i, \zeta}(\Gamma)$ is a $P B L_{r}(N)$-inner probability space.

Proof. By Lemma 4.2 to Lemma 4.11, we can get the claim immediately.

Lemma 4.13 The inner probabilistic model $P M_{\zeta}$ is a finite model.

Proof. By the definition of $S_{\zeta}$, the cardinality of $S_{\zeta}$ is no more than the cardinality of $\wp\left(S u b^{*}(\zeta)\right)$, which means $\left|S_{\zeta}\right| \leq 2^{\left|S u b^{*}(\zeta)\right|}$.

Lemma 4.14 In the canonical $P B L_{r}(N)$-model $P M_{\zeta}$, for any $\Gamma \in S_{\zeta}$ and any $\varphi \in S u b^{*}(\zeta)$, $\varphi \in \Gamma \Leftrightarrow\left(P M_{\zeta}, \Gamma\right) \models \varphi$.

Proof. We prove the lemma by induction on the structure of $\varphi$. In the following, we only prove that $B_{i}(a, \psi) \in \Gamma \Leftrightarrow\left(P M_{\zeta}, \Gamma\right) \models B_{i}(a, \psi)$.

If $B_{i}(a, \psi) \in \Gamma$, by the construction of $P M_{\zeta}, \mu_{\zeta, i, \Gamma}(X(\psi))=b \geq a$, we get $\left(P M_{\zeta}, \Gamma\right) \models B_{i}(a, \psi)$.

If $B_{i}(a, \psi) \notin \Gamma$, then $\neg B_{i}(a, \psi) \in \Gamma$, by the construction of $P M_{\zeta}$ and Lemma 4.5, since $\sup \left(\operatorname{Pro}_{\zeta, i, \Gamma}(\psi)\right) \in \operatorname{Pro}_{\zeta, i, \Gamma}(\psi)$, so we have $\sup \left(\operatorname{Pro}_{\zeta, i, \Gamma}(\psi)\right)=b<a$, and $\mu_{\zeta, i, \Gamma}(X(\psi))=b$, which implies $\left(P M_{\zeta}, \Gamma\right) \stackrel{\models}{\models} B_{i}(a, \psi)$, therefore $\left(P M_{\zeta}, \Gamma\right) \not \models B_{i}(a, \psi)$.

Proposition 4.3 (Finite model property of $P B L_{r}(N)$ ) If $\Gamma$ is a finite set of consistent formulas, then there is a finite model $P M$ such that $P M \models_{P B L_{r}(N)} \Gamma$.

Proof. By Lemma 4.14, there exists a finite $P B L_{r}(N)$-model $P M_{\wedge \Gamma}$ such that $\Gamma$ is satisfied in $P M_{\wedge \Gamma}$.

Since any inner probabilistic model of $P B L_{r}(N)$ is also an inner probabilistic model of $P B L_{r}$, and any formula of $P B L_{r}$ can be regarded as a formula of $P B L_{r}(N)$, given a consistent $P B L_{r}$-formula $\zeta$, we can construct a $P B L_{r}(N)$-inner probabilistic model $P M_{\zeta}$ that satisfies formula $\zeta$ by the above lemmas. Since $P M_{\zeta}$ is also $P B L_{r}$-inner probabilistic model, so we can construct a $P B L_{r}$ - inner probabilistic model $P M_{\zeta}$ that satisfies the given consistent $P B L_{r}$-formula $\zeta$, this implies the finite model property of $P B L_{r}$.

Proposition 4.4 (Finite model property of $P B L_{r}$ ) If $\Gamma$ is a finite set of consistent formulas, then there is a finite model $P M$ such that $P M \models P B L_{r} \Gamma$.

Proof. Let $a_{1}, a_{2}, \ldots, a_{n}$ be all rational numbers occur in the formulas in $\Gamma$. There are natural numbers $k_{1}, k_{2}, \ldots, k_{n}, N$ such that $a_{i}=k_{i} / N$. Firstly, since the axioms and rules of $P B L_{r}(N)$ is also the axioms and rules of $P B L_{r}$, therefore it is clear that if a finite set of formulas $\Gamma$ is consistent with $P B L_{r}$, then it is also consistent with $P B L_{r}(N)$. By Proposition 4.3, there is a finite model of $P B L_{r}(N), P M$, satisfying $\Gamma$. Since the model of $P B L_{r}(N)$ is also a model of $P B L_{r}$, so we get the proposition.

Proposition 4.5 (Weak completeness of $P B L_{r}$ ) If $\Gamma$ is a finite set of formulas, $\varphi$ is a formula, and $\Gamma \models_{P B L_{r}} \varphi$, then $\Gamma \vdash_{P B L_{r}} \varphi$.

Proof. Suppose not, then $(\wedge \Gamma) \wedge \neg \varphi$ is consistent with respect to $P B L_{r}$, by Proposition 4.4, there exists an inner probabilistic model $P M_{(\wedge \Gamma) \wedge \neg \varphi}$ such that $(\wedge \Gamma) \wedge \neg \varphi$ is satisfied in $P M_{(\wedge \Gamma) \wedge \neg \varphi}$, but this contradicts our assumption that $\Gamma \models_{P B L_{r}} \varphi$, thus the proposition holds.

From Proposition 4.4, we can get a procedure for checking if a formula $\varphi$ is $P B L_{r}$-consistent. We simply construct every probabilistic model with $2^{\left|S u b^{*}(\varphi)\right|}$ states (Remember that in the construction of the finite model of $\varphi$, the values of inner probability measure are in the form 
of $k / N$, where $N$ is a constant natural number. Since there are finite numbers having the form of $k / N$, where $0 \leq k \leq N$, therefore the number of inner probability measures assigned to the measurable sets is also finite, and consequently, the number of models with $2^{\left|S u b^{*}(\varphi)\right|}$ states is finite). We then check if $\varphi$ is true at some state of one of these models. By Proposition 4.4, if a formula $\varphi$ is $P B L_{r}$-consistent, then $\varphi$ is satisfiable with respect to some models. Conversely, if $\varphi$ is satisfiable with respect to some models, then $\varphi$ is $P B L_{r}$-consistent.

As a consequence, we can now show that the provability problem for $P B L_{r}$ is decidable.

Proposition 4.6 (Decidability of $P B L_{r}$ ) The provability problem for $P B L_{r}$ is decidable.

Proof. Since $\varphi$ is provable in $P B L_{r}$ iff $\neg \varphi$ is not $P B L_{r}$-consistent, we can simply check if $\neg \varphi$ is $P B L_{r}$-consistent. By the above discussion, there is a checking procedure. Hence the provability problem for $P B L_{r}$ is decidable.

\section{Comparison of Fagin and Halpern's logic with our work}

The probabilistic knowledge logic proposed by Fagin and Halpern in [3] is a famous epistemic logic with probabilistic character. In this section, we mainly compare the logic in [3] with our logics in terms of their syntax, inference system, semantics and proof technique.

1. Syntax. The basic formulas of logic in [3] can be classified into two categories: the standard knowledge logic formula such as $K_{i} \varphi$, and the probability formula such as $a_{1} w_{i}\left(\varphi_{1}\right)+\ldots+$ $a_{k} w_{i}\left(\varphi_{k}\right) \geq b$. The formula $K_{i}^{b}(\varphi)$ is an abbreviation for $K_{i}\left(w_{i}(\varphi) \geq b\right)$, intuitively, this says that "agent $i$ knows that the probability of $\varphi$ is greater than or equal to $b$ ". Except the difference of knowledge and belief operators, the formula $K_{i}^{b}(\varphi)$ is similar to the formula $B_{i}(b, \varphi)$ of this chapter. But in this chapter, $B_{i}(b, \varphi)$ is a basic formula, and there is no formula such as $a_{1} w_{i}\left(\varphi_{1}\right)+\ldots+a_{k} w_{i}\left(\varphi_{k}\right) \geq b$, because $a_{1} w_{i}\left(\varphi_{1}\right)+\ldots+a_{k} w_{i}\left(\varphi_{k}\right) \geq b$ contains non-logical symbols such as " $\times$ ", " + " and " $\geq$ ", and accordingly, the language and reasoning system have to deal with linear inequalities and probabilities. We get a tradeoff between expressive power and complexity, and the only basic formula of this chapter is $B_{i}(b, \varphi)$, which makes the syntax and axioms of our logic system simpler.

2. Inference system. The inference system in [3] consists of four components: the first component includes axioms and rules for propositional reasoning; the second component includes the standard knowledge logic; the third component allows us to reason about inequalities (so it contains axioms that allow us to deduce, for example, that $2 x \geq 2 y$ follows from $x \geq y$ ); while the fourth is the only one that has axioms and inference rules for reasoning about probability. It is worthy to note that $W 3\left(w_{i}(\varphi \wedge \psi)+w_{i}(\varphi \wedge \neg \psi)=w_{i}(\varphi)\right)$ in [3] corresponds to finite additivity, not countable infinite additivity, i.e., $\mu\left(A_{1} \cup A_{2} \cup \ldots \cup A_{n} \ldots\right)=$ $\mu\left(A_{1}\right)+\mu\left(A_{2}\right)+\ldots+\mu\left(A_{n}\right)+\ldots$, if $A_{1}, \ldots, A_{n}, \ldots$ is a countable collection of disjoint measurable sets. As Fagin and Halpern indicated, they think it is enough to introduce an axiom corresponding to finite additivity for most applications. They could not express countable infinite additivity in their language.

In this chapter, there are two components in our inference systems: the first component includes axioms and rules for propositional reasoning; the second component includes axioms and rules for probabilistic belief reasoning. In our system, when one perform reasoning, one need not to consider different kinds of axioms and rules that may involve linear inequalities or probabilities. In order to express the properties of probability (such as finite additivity, 
monotonicity or continuity) by probabilistic modal operator directly instead of by inequalities and probabilities, we introduce some new axioms and rules. While in Fagin and Halpern's paper, these properties are expressed by the axioms for linear inequalities or probabilities. Similar to Fagin and Halpern's logic system, we only express finite additivity, but not countable infinite additivity, because we cannot express such property in our language, in fact, we believe that this property cannot be expressed by finite length formula in reasoning system. On the other hand, we think the finite additivity property is enough for the most of meaningful reasoning about probabilistic belief.

3. Semantics. In [3], a Kripke structure for knowledge and probability (for $n$ agents) is a tuple $\left(S, \pi, K_{1}, \ldots, K_{n}, P\right)$, where $P$ is a probability assignment, which assigns to each agent $i \in\{1, \ldots, n\}$ and state $s \in S$ a probability space $P(i, s)=\left(S_{i, s}, X_{i, s}, \mu_{i, s}\right)$, where $S_{i, s} \subseteq S$.

To give semantics to formula such as $w_{i}(\varphi) \geq b$, the obvious way is $(M, s) \models w_{i}(\varphi) \geq b$ iff $\mu_{i, s}\left(S_{i, s}(\varphi)\right) \geq b$, here $S_{i, s}(\varphi)=\left\{s^{\prime} \in S_{i, s} \mid\left(M, s^{\prime}\right) \models \varphi\right\}$. The only problem with this definition is that the set $S_{i, s}(\varphi)$ might not be measurable (i.e., not in $\left.X_{i, s}\right)$, so that $\mu_{i, s}\left(S_{i, s}(\varphi)\right)$ might not be well defined. They considered two models. One model satisfies MEAS condition (for every formula $\varphi$, the set $S_{i, s}(\varphi) \in X_{i, s}$ ) to guarantee that this set is measurable, and the corresponding inference system $A X_{M E A S}$ has finite additivity condition $W 3$. The other model does not obey $M E A S$ condition, and the corresponding inference system $A X$ has no finite additivity condition $W 3$. To deal with the problem in this case, they adopted the inner measures $\left(\mu_{i, s}\right)^{*}$ rather than $\mu_{i, s}$, here $\left(\mu_{i, s}\right)^{*}(A)=\sup \left(\left\{\mu_{i, s}(B) \mid B \subseteq A\right.\right.$ and $\left.\left.B \in X\right\}\right)$, here $\sup (A)$ is the least upper bound of $A$. Thus, $(M, s) \models w_{i}(\varphi) \geq b$ iff $\left(\mu_{i, s}\right)^{*}\left(S_{i, s}(\varphi)\right) \geq b$.

Similar to the model of $A X_{M E A S}$ in [3], in the model of $P B L_{\omega}, X_{i, s}$ satisfies the following conditions: (a) If $p$ is an atomic formula, then $\operatorname{ev}_{P M}(p)=\left\{s^{\prime} \mid \pi\left(s^{\prime}, p\right)=\right.$ true $\} \in X_{i, s}$; (b) If $A \in X_{i, s}$, then $S_{i, s}-A \in X_{i, s}$; (c) If $A_{1}, A_{2} \in X_{i, s}$, then $A_{1} \cap A_{2} \in X_{i, s}$; (d) If $A \in X_{i, s}$ and $a \in[0,1]$, then $\left\{s^{\prime} \mid \mu_{i, s^{\prime}}(A) \geq a\right\} \in X_{i, s}$. From these conditions, we can prove by structural induction that for every formula $\varphi$, the set $e v_{P M}(\varphi) \in X_{i, s}$. Therefore, the model of $P B L_{\omega}$ also satisfies the condition $M E A S$. Moreover, similar to the model of $A X_{M E A S}$, probability measure in the model of $P B L_{\omega}$ satisfies finite additivity property.

In contrast with $P B L_{\omega}$, the models of $P B L_{f}$ and $P B L_{r}$ are similar to the model of $A X$ in [3]. There is an inner probability measure rather than probability measure in the models of $P B L_{f}$ and $P B L_{r}$. In the model of $A X$, the semantics of formula is given by inner probability measure induced by probability measure. Meanwhile, in the models of $P B L_{f}$ and $P B L_{r}$, we introduce inner probability measure directly, which satisfies some weaker additivity properties.

Since there is no accessible relation in our model, we need not to consider the conditions about accessible relations. The only conditions we have to consider are probability space at different states, which simplifies the description and construction of model.

4. Proof technique of completeness. In [3], they prove the completeness by reducing the problem to the existence of solution of a finite set of linear inequalities. But this method does not provide the value of measure assigned to every possible world, and just assures the existence of measure. Moreover, this method cannot provide completeness property in the case of infinite set of formulas, which needs some linear inequalities axioms to characterize the existence of solutions of infinitely many linear inequalities that contain infinitely many variables. This seems impossible when we have only finite-length formulas in the language. In this chapter, the proof for completeness is significant different from the proof in [3]. There 
are no auxiliary axioms such as the probability axioms and linear inequality axioms, which are necessary in the proof of [3]. We prove the completeness by constructing the model that satisfies the given consistent formulas set, our proof can also be used to deal with the case of infinite set of formulas. Furthermore, our proof can be generalized to get the completeness of other probabilistic logic systems because it depends very lightly on the concrete axioms and rules.

\section{Conclusions}

In this chapter, we proposed probabilistic belief logics $P B L_{\omega}, P B L_{f}$ and $P B L_{r}$, and gave the respective probabilistic semantics of these logics. Furthermore we proved the soundness and completeness of $P B L_{\omega}$, the finite model property of $P B L_{f}$ and the decidability of $P B L_{r}$. The above probabilistic belief logics allow the reasoning of uncertain information of agent in artificial intelligent systems.

The probabilistic semantics of probabilistic belief logic can also be applied to describe other probabilistic modal logic by adding the respective restricted conditions on probability space. Just as different assumptions about the relationship between worlds, can be captured with different axioms in modal logics, different assumptions about the interrelationships between probability assignment spaces at different states, can also be captured axiomatically. Furthermore, the completeness proof in this chapter can be applied to prove the completeness of other probabilistic modal logics.

It seems to us that some further research directions lie in the following several problems: whether the finite model property for $P B L_{\omega}$ holds, whether the decidability for the provability problem of $P B L_{\omega}$ or $P B L_{f}$ holds, moreover, if the decidability holds, what is the complexity of the corresponding provability problem. These problems seem to be much more difficult and remain open. The techniques used in classical modal logics are not suit to solve such problems, and some new techniques may be necessary.

\section{Acknowledgment}

This work was supported by the National Natural Science Foundation of China under Grants No. 60873025, and the Foundation of Provincial Key Laboratory for Computer Information Processing Technology of Soochow University under Grant No. KJS0920.

\section{References}

[1] R. J. Aumann. Agreeing to disagree. The Annals of Statistics, 1976, 4(6): 1236-1239.

[2] F. Bacchus. Representing and reasoning with probabilistic knowledge: a logical approach to probabilities. Cambridge, Mass. : MIT Press, 1990.

[3] R. Fagin and J. Y. Halpern. Reasoning about knowledge and probability. J ACM, 1994, 41(2): 340-367.

[4] R. Fagin, J. Y. Halpern and N. Megiddo. A logic for reasoning about probabilities, Information and Computation. 1990, 87(1/2): 78-128.

[5] R. Fagin, J. Y. Halpern, Y. Moses and M. Y.Vardi. Reasoning about knowledge. Cambridge, Massachusetts: The MIT Press, 1995. /2): 78-128.

[6] M. Fattorosi-Barnaba and G. Amati. Modal Operators with Probabilistic Interpretations i. Studia Logica, 1987, XLVI(4):383-393. 
[7] Y. Feldman. A decidable prepositional probabilistic dynamic logic with explicit probabilities. Information and Control, 1984, 63: 11-38.

[8] D. Fudenberg and J. Tirole. Game theory. Cambridge, Massachusetts: The MIT Press, 1991.

[9] D. Gillies. Philosophical Theories of Probability, Philosophical issues in science, London and New York: Routledge, 2000.

[10] J. Y. Halpern. The relationship between knowledge, belief, and certainty. Annals of Mathematics and Artificial Intelligence 1991, 4: 301-322.

[11] J. Y. Halpern. Lexicographic probability, conditional probability, and nonstandard probability. In Proceedings of the Eighth Conference on Theoretical Aspects of Rationality and Knowledge, 2001, 17-30.

[12] J. Y. Halpern and Y. Moses. Knowledge and common knowledge in a distributed environment. J ACM, 1990, 37(3): 549-587.

[13] J. Y. Halpern and M. R. Tuttle. Knowledge, probability, and adversaries,. J.ACM, 1993, 40(4): 917-962.

[14] S. Hart and M. Sharir. Probabilistic temporal logics for finite and bounded models. In Proceedings of the 16th ACM Symposium on Theory of Computing, 1984, ACM, New York, 1-13.

[15] J. Hintikka. Knowledge and belief. Ithaca, NY: Cornell University Press, 1962.

[16] W. van der Hoek. Some considerations on the logic PFD: A logic combining modality and probability. J. Applied Non-Classical Logics, 7(3):287-307, 1997.

[17] H. J. Keisler. Probability quantifiers, in J. Barwise and S. Feferman, Eds. Model-theoretical logics, 509-556, Springer-Verlag, New York/Berlin, 1985.

[18] B. P. Kooi. Probabilistic Dynamic Epistemic Logic. Journal of Logic, Language and Information 2003, 12: 381-408.

[19] S. Kripke. A semantics analysis of modal logic, I: Normal modal propositional calculi. Z. Math. Logik Grundl. Math, 1963, 9: 67-96.

[20] A. Laux and H. Wansing. (eds) Knowledge and belief in philosophy and artificial intelligence, Akademie Verlag GmbH, Berlin, 1995.

[21] T. Lukasiewicz. Weak nonmonotonic probabilistic logics, KR2004, Whistler, Canada, 2004.

[22] B. Milch and D. Koller. Probabilistic Models for Agent's Beliefs and Decisions. Proc. 16th Conference on Uncertainty in Artificial Intelligence 2000: 389-396.

[23] J. Pearl. Probabilistic reasoning in intelligent systems: Networks of plausible inference. Morgan Kaufmann, San Mateo, CA, USA, 1988.

[24] A. S. Rao and M. P. Georgeff. Modeling rational agents within a BDI-architecture. Proceeding of KR-91, 1991, San Mateo, CA, USA, 473-484.

[25] G. Shafer. A mathematical theory of evidence. Princeton University Press, Princeton, N.J, 1976. 


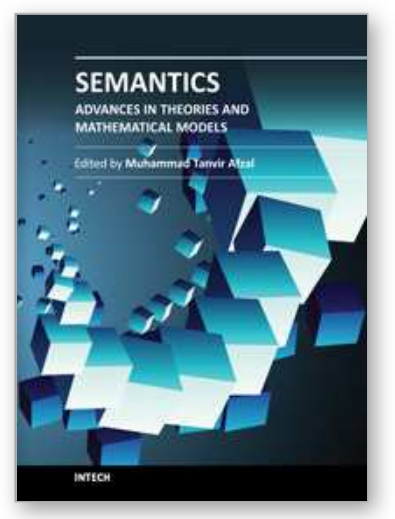

\author{
Semantics - Advances in Theories and Mathematical Models \\ Edited by Dr. Muhammad Tanvir Afzal
}

ISBN 978-953-51-0535-0

Hard cover, 284 pages

Publisher InTech

Published online 25, April, 2012

Published in print edition April, 2012

The current book is a nice blend of number of great ideas, theories, mathematical models, and practical systems in the domain of Semantics. The book has been divided into two volumes. The current one is the first volume which highlights the advances in theories and mathematical models in the domain of Semantics. This volume has been divided into four sections and ten chapters. The sections include: 1) Background, 2) Queries, Predicates, and Semantic Cache, 3) Algorithms and Logic Programming, and 4) Semantic Web and Interfaces. Authors across the World have contributed to debate on state-of-the-art systems, theories, mathematical models in the domain of Semantics. Subsequently, new theories, mathematical models, and systems have been proposed, developed, and evaluated.

\title{
How to reference
}

In order to correctly reference this scholarly work, feel free to copy and paste the following:

Zining Cao (2012). Probabilistic Belief Logics for Uncertain Agents, Semantics - Advances in Theories and Mathematical Models, Dr. Muhammad Tanvir Afzal (Ed.), ISBN: 978-953-51-0535-0, InTech, Available from: http://www.intechopen.com/books/semantics-advances-in-theories-and-mathematical-models/probabilisticbelief-logics-for-uncertain-agents

\section{INTECH}

open science | open minds

\section{InTech Europe}

University Campus STeP Ri

Slavka Krautzeka 83/A

51000 Rijeka, Croatia

Phone: +385 (51) 770447

Fax: +385 (51) 686166

www.intechopen.com

\section{InTech China}

Unit 405, Office Block, Hotel Equatorial Shanghai

No.65, Yan An Road (West), Shanghai, 200040, China

中国上海市延安西路65号上海国际贵都大饭店办公楼 405 单元

Phone: +86-21-62489820

Fax: +86-21-62489821 
(C) 2012 The Author(s). Licensee IntechOpen. This is an open access article distributed under the terms of the Creative Commons Attribution 3.0 License, which permits unrestricted use, distribution, and reproduction in any medium, provided the original work is properly cited. 